J. Appl. Numer. Optim. 3 (2021), No. 2, pp. 243-261

Available online at http://jano.biemdas.com

https://doi.org/10.23952/jano.3.2021.2.02

\title{
A SHAPE DERIVATIVE FOR OPTIMAL CONTROL OF THE NONLINEAR BRINKMAN-FORCHHEIMER EQUATION
}

\author{
JOSÉ RODRIGO GONZÁLEZ GRANADA ${ }^{1}$, VICTOR A. KOVTUNENKO2,3,** \\ ${ }^{1}$ Department of Mathematics, Universidad Tecnológica de Pereira, 660003 Pereira, Colombia \\ ${ }^{2}$ Institute for Mathematics and Scientific Computing, \\ Karl-Franzens University of Graz, NAWI Graz, Heinrichstr.36, 8010 Graz, Austria \\ ${ }^{3}$ Lavrentyev Institute of Hydrodynamics, \\ Siberian Division of the Russian Academy of Sciences, 630090 Novosibirsk, Russia
}

\begin{abstract}
For a generalized Brinkman-Forchheimer's equation under divergence-free and mixed boundary conditions, the stationary equilibrium problem and the inverse problem of shape optimal control are considered. For a convex, geometry-dependent objective function, the equilibrium-constrained optimization is treated with the help of an adjoint state within the Lagrange approach. The shape differentiability of a Lagrangian with respect to linearized shape perturbations is derived in the analytic form by the velocity method. A Hadamard representation of the shape derivative using boundary integrals is derived. Its applications to path-independent integrals and to the gradient descent method are illustrated.
\end{abstract}

Keywords. Incompressible flow; Semilinear equation; Equilibrium-constrained optimization; Optimal control; Shape derivative.

\section{INTRODUCTION}

The current paper is devoted to the shape differentiablity of geometry-dependent objective functions, as they are considered in shape optimal control. The optimal value objective is subject to the nonlinear equilibrium described by generalized Brinkman-Forchheimer's equation under the incompressibility and mixed Dirichlet-Neumann boundary conditions. The nonlinear boundary value problem describes the single-phase fluid flow in a porous medium [1]. The topic under consideration belongs to the fields of shape optimization, optimal control and inverse problems. These problems are ill-posed, since objectives have typically many local minima when perturbing equilibrium. Therefore, our theoretical result is of practical importance since it provides robust numerical methods of iterative optimization, namely, the shape derivative for the gradient descent method.

We cite textbooks [2,3] for the general theory of shape optimization, [4] for mathematical programs with equilibrium constraints (MPEC), and [5, 6, 7] for parameter identification in (hemi-) variational inequalities. For numerical treatment of optimal control problems, we refer

\footnotetext{
${ }^{*}$ Corresponding author.

E-mail addresses: jorodryy @utp.edu.co (J.R. González Granada), victor.kovtunenko@uni-graz.at (V.A. Kovtunenko).

Received July 31, 2020; Accepted November 3, 2020.
}

(C)2021 Journal of Applied and Numerical Optimization 
to $[8,9,10]$. For relevant applications in mathematical physics, we refer to $[11,12,13,14]$. The shape derivative is important since it determines the optimality condition with respect to geometry perturbations. An abstract formalism of directional differentiability for saddle-point optimal value functions was established in [15] by using adjoints states within the Lagrange approach. Further extensions to shape differentiability were done in [2], and to constrained PDE models in $[16,17,18]$ and other works. With the help of the Lagrange approach, previously we derived the shape derivative for nonpenetrating cracks [19, 20, 21, 22]. It was employed for fracture analysis by Griffith's formula and optimal control of cracks [23, 24, 25, 26], for breaking-line identification under equilibrium constraints [27]. In this respect, the model under consideration can be interpreted within the incompressible elasticity in solid mechanics.

In the context of fluid mechanics described by Stokes and Navier-Stokes equations, we refer to [28] for the mathematical theory of incompressible flows, to [29, 30] for flows in porous media, and in thin layers in [31]. See the related results on optimal control of fluids [32], on free boundary problems [33], and on the coefficient identification based on least squares [34, 35]. The shape optimization approach for compressible Navier-Stokes equations was developed in [36]. Recently, we studied the shape differentiability of objectives subject to the divergence-free (incompressible) equilibrium described by Stokes [37] and Brinkman equations [38]. The both models are liner ones. In the current paper we investigate generalized Brinkman-Forchheimer's model implying a semilinear elliptic equation, see the well-posedness analysis [39, 40, 41] and the integral potential method [42].

The main difficulty concerns the nonlinearity of equilibrium equations, which does not allow to apply the standard Lagrange approach. For this reason, we linearize the perturbed Lagrangian at the reference (unperturbed) state. For the respective theory employing associated to adjoint operators we refer the readers to [43]. With its help we prove rigorously the shape derivative of the optimal value objective subject to Brinkman-Forchheimer's state equation and using its adjoint state. The analytical expression of the shape derivative and the respective Hadamard representation are obtained, which are advantageous for the gradient descent algorithm solving the inverse problem of shape optimal control.

In Section 2, we establish well-posedness of the forward Brinkman-Forchheimer problem in Theorem 2.1 and formulate the inverse problem. In Section 3, an equivalent saddle-point formulation using the adjoint state is given in Theorem 3.1. Based on Lemmas 4.1 and 4.2, the main Theorem 4.1 on the shape derivative of the Lagrangian with respect to linearized perturbations is proved in Section 4. In Section 5, the Hadamard formula is established in Theorem 5.1, its application to path-independent integrals is presented in Corollaries 5.1 and 5.2, and to a descent direction in Corollary 5.3.

\section{FORWARD AND INVERSE NONLINEAR BRINKMAN-FORCHHEIMER'S PROBLEMS}

For a family of parameter-dependent Lipschitz domains, we provide results on existence of variational solutions to the Brinkman-Forchheimer equation. Thereafter, an optimal control problem is introduced where the control is given by the parameter that describes geometric domains on which the equilibrium equation is solved.

We start with a family of parameter-dependent geometries in a bounded hold-all set $D$ :

$$
\left[t \mapsto \Omega_{t}\right]:\left(t_{0}, t_{1}\right) \mapsto D, \quad D \subset \mathbb{R}^{d}, \quad d=2,3 .
$$


For every fixed kinematic parameter $t$, let a domain $\Omega_{t}$ in (2.1) have the Lipschitz boundary $\partial \Omega_{t}$ with the unit normal vector $n^{t}=\left(n_{1}^{t}, \ldots, n_{d}^{t}\right)^{\top}$ outward to $\Omega_{t}$. The upper script ${ }^{\top}$ swaps between rows and columns. We assume that $\partial \Omega_{t}$ consists of nonempty, disjoint parts $\Gamma_{t}^{\mathrm{D}}$ and $\Gamma_{t}^{\mathrm{N}}$.

Given stationary force $f(x)=\left(f_{1}, \ldots, f_{d}\right)^{\top} \in H^{1}(D)^{d}$, we consider generalized (with respect to a growth exponent $m$ ) Brinkman-Forchheimer's equation under the mixed DirichletNeumann boundary conditions: find a flow velocity vector $u^{t}(x)=\left(u_{1}^{t}, \ldots, u_{d}^{t}\right)^{\top}$ and a pressure $p_{t}(x)$ satisfying the following relations:

$$
\begin{gathered}
-\mu \Delta u^{t}+\alpha\left|u^{t}\right|^{m-2} u^{t}+\nabla p_{t}=f, \quad \operatorname{div}\left(u^{t}\right)=0 \quad \text { in } \Omega_{t}, \\
\varepsilon\left(u^{t}\right):=\frac{1}{2}\left(\nabla u^{t}+\left(\nabla u^{t}\right)^{\top}\right), \\
u^{t}=0 \quad \text { on } \Gamma_{t}^{\mathrm{D}}, \quad-2 \mu \varepsilon\left(u^{t}\right) n^{t}+p_{t} n^{t}=0 \quad \text { on } \Gamma_{t}^{\mathrm{N}} .
\end{gathered}
$$

This system describes steady, slow flows of fluids through porous media. In (2.2a), the former equation comes from the balance of linear momentum (see $[39,41])$, and the latter one implies incompressibility condition. Here the fluid viscosity $\mu>0$, the drag coefficient $\alpha \geq 0$, and the growth exponent $m>1$ guarantees well-posedness (see Theorem 2.1). In particular cases, $m=3$ implies the classic, quadratic Forchheimer law, whereas the drag term reduces to the linear Darcy law when $m=2$. For other than (2.2c) boundary conditions we refer to [35, 40].

The conventional notation in (2.2) stands for the gradient vector $\nabla:=\left(\partial / \partial x_{1}, \ldots, \partial / \partial x_{d}\right)^{\top}$, the divergence $\operatorname{div}:=\operatorname{tr}(\nabla)$, the Laplace operator $\Delta=\operatorname{div}(\nabla)$. The gradient of a vector is defined as $\nabla u^{t}=\left(\partial u_{i}^{t} / \partial x_{j}\right)_{i, j=1}^{d}$, the linearized strain $\varepsilon\left(u^{t}\right)$ in (2.2b) is a $d$-by- $d$ symmetric matrix, and $\varepsilon\left(u^{t}\right) n^{t}$ in (2.2c) implies the matrix-vector multiplication. Accounting the no-slip boundary condition in (2.2c) (the former one) forces Sobolev's function space for admissible velocities:

$$
V\left(\Omega_{t}\right):=\left\{w=\left(w_{1}, \ldots, w_{d}\right)^{\top} \in H^{1}\left(\Omega_{t}\right)^{d} \mid \quad w=0 \text { a.e. } \Gamma_{t}^{\mathrm{D}}\right\},
$$

and the incompressibility condition in (2.2a) is determined well by the mapping

$$
[w \mapsto \operatorname{div}]: V\left(\Omega_{t}\right) \mapsto L^{2}\left(\Omega_{t}\right) .
$$

Theorem 2.1 (Well-posedness). For $m \in\left(1, m_{0}\right), m_{0}>1$, where $m_{0}<6$ in $3 d$ and arbitrary $m_{0}<\infty$ in $2 d$, there exists a solution pair $\left(u^{t}, p_{t}\right) \in V\left(\Omega_{t}\right) \times L^{2}\left(\Omega_{t}\right)=: U\left(\Omega_{t}\right)$ satisfying the nonlinear Brinkman-Forchheimer problem (2.2) in a mixed variational form:

$$
\begin{gathered}
\int_{\Omega_{t}}\left(2 \mu \varepsilon\left(u^{t}\right) \cdot \varepsilon(w)+\alpha\left|u^{t}\right|^{m-2}\left(u^{t}\right)^{\top} w-p_{t} \operatorname{div}(w)\right) d x=\int_{\Omega_{t}} f^{\top} w d x, \\
\int_{\Omega_{t}} \lambda \operatorname{div}\left(u^{t}\right) d x=0 \quad \text { for all }(w, \lambda) \in U\left(\Omega_{t}\right),
\end{gathered}
$$

where the dot in (2.5a) denotes the scalar product of second order tensors. If $m \geq 2$, then the solution pair is unique.

Proof. We can rewrite the equilibrium equation in (2.2a) equivalently with the help of equality

$$
-\mu \Delta u^{t}=-\mu\left(\Delta u^{t}+\nabla \operatorname{div}\left(u^{t}\right)\right)=-2 \mu \operatorname{div} \varepsilon\left(u^{t}\right)
$$

due to incompressibility and the strain tensor in (2.2b). Then the weak formulation (2.5) is derived by multiplying equations (2.2a) with the corresponding test functions $w, \lambda$, integrating them over $\Omega_{t}$, and using boundary conditions (2.2c) after integration by parts. 
The first term in (2.5a) determines a bounded, symmetric, bilinear quadratic form, which is strongly elliptic by the Korn-Poincaré inequality:

$$
\int_{\Omega_{t}} \varepsilon(w) \cdot \varepsilon(w) d x \geq K_{\mathrm{KP}}\|w\|_{H^{1}\left(\Omega_{t}\right)^{d}}^{2} \quad \text { for } w \in V\left(\Omega_{t}\right), \quad K_{\mathrm{KP}}>0 .
$$

By the continuous, compact Sobolev's embedding $H^{1}\left(\Omega_{t}\right) \subset L^{m}\left(\Omega_{t}\right)$ as $m \in(1,2 d /(d-2))$, we have the uniform estimate

$$
\int_{\Omega_{t}}|w|^{m} d x \leq K_{m}\|w\|_{H^{1}\left(\Omega_{t}\right)^{d}}^{m} \quad \text { for } w \in H^{1}\left(\Omega_{t}\right)^{d}, \quad K_{m}>0 .
$$

Henceforth, applying (2.8) and the Hölder inequality, the second (nonlinear) term in (2.5a) admits the upper bound for $u, w \in H^{1}\left(\Omega_{t}\right)$ :

$$
\left.\left|\int_{\Omega_{t}}\right| u\right|^{m-2} u^{\top} w d x \mid \leq\|u\|_{L^{m}\left(\Omega_{t}\right)^{d}}^{m-1}\|w\|_{L^{m}\left(\Omega_{t}\right)^{d}} \leq K_{m}^{1 / m}\|u\|_{L^{m}\left(\Omega_{t}\right)^{d}}^{m-1}\|w\|_{H^{1}\left(\Omega_{t}\right)^{d}} .
$$

For the third term in (2.5a), the inf-sup (LBB) condition holds since $\Gamma_{t}^{\mathrm{N}} \neq \emptyset$ (see [28]):

$$
\sup _{w \in V\left(\Omega_{t}\right), w \neq 0} \frac{1}{\|w\|_{H^{1}\left(\Omega_{t}\right)^{d}}} \int_{\Omega_{t}} \lambda \operatorname{div}(w) d x \geq K_{\mathrm{LBB}}\|\lambda\|_{L^{2}\left(\Omega_{t}\right)} \text { for } \lambda \in L^{2}\left(\Omega_{t}\right), K_{\mathrm{LBB}}>0 .
$$

Then the mapping in (2.4) is surjective. Further we employ fixpoint arguments [39, 41].

In subspaces $V^{n}\left(\Omega_{t}\right)=\operatorname{span}\left\{\phi^{1}, \ldots, \phi^{n}\right\}$ and $H_{n}\left(\Omega_{t}\right)=\operatorname{span}\left\{\psi_{1}, \ldots, \psi_{n}\right\}$ of finite dimension $n \in \mathbb{N}$, which are spanned by orthogonal bases $\left(\phi^{k}\right)_{k \in \mathbb{N}} \in V\left(\Omega_{t}\right)$ and $\left(\psi_{k}\right)_{k \in \mathbb{N}} \in L^{2}\left(\Omega_{t}\right)$, we set the conforming Galerkin approximation of (2.5) by the nonlinear equations:

$$
\begin{gathered}
\int_{\Omega_{t}}\left(2 \mu \varepsilon\left(u^{n}\right) \cdot \varepsilon(w)+\alpha\left|u^{n}\right|^{m-2}\left(u^{n}\right)^{\top} w-p_{n} \operatorname{div}(w)\right) d x=\int_{\Omega_{t}} f^{\top} w d x, \\
\int_{\Omega_{t}} \lambda \operatorname{div}\left(u^{n}\right) d x=0 \quad \text { for all }(w, \lambda) \in V^{n}\left(\Omega_{t}\right) \times H_{n}\left(\Omega_{t}\right) .
\end{gathered}
$$

Testing (2.11a) with $w=u^{n}$, and using $\operatorname{div}\left(u^{n}\right)=0$ and (2.7), we derive the upper bound

$$
2 \mu K_{\mathrm{KP}}\left\|u^{n}\right\|_{H^{1}\left(\Omega_{t}\right)^{d}}^{2}+\alpha \int_{\Omega_{t}}\left|u^{n}\right|^{m} d x \leq\|f\|_{L^{2}\left(\Omega_{t}\right)^{d}}\left\|u^{n}\right\|_{H^{1}\left(\Omega_{t}\right)^{d}},
$$

and subsequently we estimate

$$
2 \mu K_{\mathrm{KP}}\left\|u^{n}\right\|_{H^{1}\left(\Omega_{t}\right)^{d}} \leq\|f\|_{L^{2}\left(\Omega_{t}\right)^{d}}=: K_{u}, \quad\left\|u^{n}\right\|_{L^{m}\left(\Omega_{t}\right)^{d}}^{m} \leq \frac{K_{u}^{2}}{2 \mu \alpha K_{\mathrm{KP}}} .
$$

Rearranging (2.11a), dividing it by the norm $\|w\|_{H^{1}\left(\Omega_{t}\right)^{d}}$ and taking supremum over $w \in V\left(\Omega_{t}\right)$ for $w \neq 0$, it follows from (2.9), (2.10), (2.12a) the uniform estimate

$$
\begin{aligned}
& K_{\mathrm{LBB}}\left\|p_{n}\right\|_{L^{2}\left(\Omega_{t}\right)} \leq \sup _{w \in V\left(\Omega_{t}\right), w \neq 0} \frac{1}{\|w\|_{H^{1}\left(\Omega_{t}\right)^{d}}} \int_{\Omega_{t}} p_{n} \operatorname{div}(w) d x \\
= & \sup _{w \in V\left(\Omega_{t}\right), w \neq 0} \frac{1}{\|w\|_{H^{1}\left(\Omega_{t}\right)^{d}}} \int_{\Omega_{t}}\left(2 \mu \varepsilon\left(u^{n}\right) \cdot \varepsilon(w)+\left(\alpha\left|u^{n}\right|^{m-2} u^{n}-f\right)^{\top} w\right) d x \leq 2 \mu\left\|u^{n}\right\|_{H^{1}\left(\Omega_{t}\right)^{d}} \\
+ & \alpha K_{m}^{1 / m}\left\|u^{n}\right\|_{L^{m}\left(\Omega_{t}\right)^{d}}^{m-1}+\|f\|_{L^{2}\left(\Omega_{t}\right)^{d}} \leq \frac{K_{u}}{K_{\mathrm{KP}}}+\left(\alpha K_{m}\right)^{1 / m}\left(\frac{K_{u}^{2}}{2 \mu K_{\mathrm{KP}}}\right)^{(m-1) / m}+K_{u}=: K_{\lambda} . \quad(2.12 \mathrm{~b})
\end{aligned}
$$


Since the strong and weak convergence coincide on finite-dimensional spaces, according to (2.12), the operator of the problem (2.11) is coercive and sequentially continuous in the ball

$$
B:=\left\{(w, \lambda) \in V^{n}\left(\Omega_{t}\right) \times H_{n}\left(\Omega_{t}\right) \mid \quad\|w\|_{H^{1}\left(\Omega_{t}\right)^{d}} \leq \frac{K_{u}}{2 \mu K_{\mathrm{KP}}},\|\lambda\|_{L^{2}\left(\Omega_{t}\right)} \leq \frac{K_{\lambda}}{K_{\mathrm{LBB}}}\right\} .
$$

Then a fixed point $\left(u^{n}, p_{n}\right) \in V^{n}\left(\Omega_{t}\right) \times H_{n}\left(\Omega_{t}\right)$ solving (2.11) exists by Brouwer's theorem.

From the a-priori estimates (2.12), we conclude with a subsequence of integers $n_{k} \rightarrow \infty$ and an accumulation point $\left(u^{t}, p_{t}\right) \in V\left(\Omega_{t}\right) \times L^{2}\left(\Omega_{t}\right)$ such that

$$
\left(u^{n_{k}}, p_{n_{k}}\right) \rightarrow\left(u^{t}, p_{t}\right) \text { weakly in } V\left(\Omega_{t}\right) \times L^{2}\left(\Omega_{t}\right), \quad u^{n_{k}} \rightarrow u^{t} \text { strongly in } L^{m}\left(\Omega_{t}\right)^{d},
$$

where the strong convergence in (2.14) is due to the compact embedding $H^{1}\left(\Omega_{t}\right) \subset L^{m}\left(\Omega_{t}\right)$. On taking the limit as $n_{k} \rightarrow \infty$ in (2.11), with the help of (2.14), we get variational problem (2.5).

The nonlinear term in (2.5a) is monotone for $m \geq 2$ because of the representation:

$$
\left(|w|^{m-2} w-|u|^{m-2} u\right)^{\top}(w-u)=\frac{1}{2}\left(|w|^{m-2}+|u|^{m-2}\right)|w-u|^{2}+\frac{1}{2}\left(|w|^{m-2}-|u|^{m-2}\right)\left(|w|^{2}-|u|^{2}\right),
$$

then the velocity $u^{t}$ solving (2.5) is unique. In this case, the LBB condition (2.10) guarantees the uniqueness of pressure $p_{t}$. The proof is complete.

Let a set $O_{t} \subset \overline{\Omega_{t}}$ of the Hausdorff measure $d \mu_{O_{t}}$ be either a subdomain with $d \mu_{O_{t}}=d x$, or an oriented Lipschitz manifold of codimension one and the measure $d \mu_{O_{t}}=d S_{x}$. The corresponding restriction $w \in H^{1}\left(O_{t}\right)^{d}$, or trace $w \in H^{1 / 2}\left(O_{t}\right)^{d}$ associating an observation is surjective for $w \in V\left(\Omega_{t}\right)$. We consider an objective function based on fidelity observations:

$$
\mathscr{J}\left(w ; O_{t}\right):=\int_{O_{t}} \gamma(w) d \mu_{O_{t}}, \quad \gamma(w) \in L^{1}(D), \quad \text { for } w \in V\left(\Omega_{t}\right) .
$$

The typical example is the least-square misfit from a measurement $z \in L^{2}\left(O_{t}\right)$ (see $\left.[11,13,27]\right)$ :

$$
\gamma(w)(x)=\frac{1}{2} \rho(x)|w-z|^{2}, \quad \rho \in C^{1}(D), \quad \rho \geq 0 .
$$

With the help of (2.15), we introduce an inverse problem of the optimal shape control.

Induced by Brinkman-Forchheimer's flow, we aim to find an optimal shape $\Omega_{*} \subset D$ such that

$$
\min _{\Omega_{t} \subset D}\left\{j(0):=\mathscr{J}\left(u^{t} ; O_{t}\right) \quad \text { for }\left(u^{t}, p_{t}\right) \in U\left(\Omega_{t}\right) \text { solving }(2.5)\right\} .
$$

In order to solve (2.17) by gradient methods of numerical optimization, this needs a descent direction such that $\partial_{+} j(0)<0$ for a directional derivative at $s=0$ (if exists):

$$
\partial_{+} j(0):=\lim _{s \rightarrow 0^{+}} \frac{j(s)-j(0)}{s} \quad \text { (one sided). }
$$

In the following, we construct proper perturbations of problem (2.5) providing us with a differentiable optimal value objective function $j(s): I \mapsto \overline{\mathbb{R}}$ in (2.18). 


\section{LINEARIZED PERTURBATIONS AND LAGRANGE SADDLE-POINT FORMULATION}

The shape functional under consideration is a $L^{2}$-tracking type functional, which is known to be ill-posed. Therefore, we apply the Lagrangian approach to the equilibrium-constrained optimal control problem, and we construct perturbations of the state linearized at the solution $u^{t}$. We begin with shape perturbations of the domain by using the velocity method.

For small perturbation parameters $s \in I:=\left[0, s_{0}\right), s_{0} \in\left(0, t_{1}-t\right)$, kinematic flows

$$
\left[(s, x) \mapsto \phi_{s}\right],\left[(s, y) \mapsto \phi_{s}^{-1}\right] \in C^{1}\left(I ; W^{1, \infty}(\bar{D})\right)^{d}
$$

associate a coordinate transformation $y=\phi_{s}(x)$ and its inverse $x=\phi_{s}^{-1}(y)$ satisfying the identities $\left[\phi_{s}^{-1} \circ \phi_{S}\right](x)=x$ and $\left[\phi_{S} \circ \phi_{s}^{-1}\right](y)=y$. We suppose that it forms a diffeomorphism:

$$
x \mapsto \phi_{s}:\left(\Omega_{t}, \Gamma_{t}^{\mathrm{D}}, \Gamma_{t}^{\mathrm{N}}, O_{t}\right) \mapsto\left(\Omega_{t+s}, \Gamma_{t+s}^{\mathrm{D}}, \Gamma_{t+s}^{\mathrm{N}}, O_{t+s}\right) .
$$

The kinematic velocity $\Lambda(t, x) \in C\left(\left[t_{0}, t_{1}\right] ; W^{1, \infty}(\bar{D})\right)^{d}$ is supposed from (3.1) by the formula

$$
\Lambda(t+s, y):=\frac{d}{d s} \phi_{s}\left(\phi_{s}^{-1}(y)\right) .
$$

Conversely, given explicitly a kinematic velocity vector-field

$$
\Lambda=\left(\Lambda_{1}, \ldots, \Lambda_{d}\right)^{\top}(t, x) \in C\left(\left[t_{0}, t_{1}\right] ; W^{1, \infty}(\bar{D})\right)^{d},\left.\quad \Lambda\right|_{\partial D}=0,
$$

where the last condition preservs the hold-all domain $D$, it determines the flows in (3.1) as a solution vector $\phi_{s}=\left(\left(\phi_{s}\right)_{1}, \ldots,\left(\phi_{s}\right)_{d}\right)^{\top}$ to the non-autonomous ODE system (see [16, 22]):

$$
\frac{d}{d s} \phi_{s}=\Lambda\left(t+s, \phi_{s}\right) \text { for } s \in I, \quad \phi_{s}=x \text { as } s=0,
$$

and $\phi_{s}^{-1}(y)=\left(\left(\phi_{s}^{-1}\right)_{1}, \ldots,\left(\phi_{s}^{-1}\right)_{d}\right)^{\top}$ to the transport equation:

$$
\frac{\partial}{\partial s} \phi_{s}^{-1}+\left.\left(\nabla_{y} \phi_{s}^{-1}\right) \Lambda\right|_{t+s}=0 \text { in } I \times D, \quad \phi_{s}^{-1}=y \text { as } s=0 .
$$

In (3.5b) the second order tensor $\nabla_{y} \phi_{s}^{-1}=\left(\partial\left(\phi_{s}^{-1}\right)_{i} / \partial y_{j}\right)_{i, j=1}^{d}$, and notation $\left.\Lambda\right|_{t+s}=\Lambda(t+s, y)$.

The diffeomorphism (3.2) provides bijectivity between the function spaces:

$$
\left[w \mapsto w \circ \phi_{s}^{-1}\right]:\left(V\left(\Omega_{t}\right), L^{2}\left(\Omega_{t}\right), L^{1}\left(O_{t}\right)\right) \mapsto\left(V\left(\Omega_{t+s}\right), L^{2}\left(\Omega_{t+s}\right), L^{1}\left(O_{t+s}\right)\right) .
$$

With the help of (3.6), we transform the perturbed objective $\mathscr{J}\left(\tilde{w} ; O_{t+s}\right)=\int_{O_{t+s}} \gamma(\tilde{w}) d \mu_{O_{t+s}}$ given according to (2.15) by $\gamma(\tilde{w})$ for functions $\tilde{w} \in V\left(\Omega_{t+s}\right)$ such that

$$
\gamma\left(w \circ \phi_{s}^{-1}\right)\left(\phi_{s}(x)\right)=: \tilde{\gamma}_{s}(w)(x), \quad \mathscr{J}\left(w \circ \phi_{s}^{-1} ; O_{t+s}\right)=: \tilde{J}\left(s, w ; O_{t}\right)=\int_{O_{t}} \tilde{\gamma}_{s}(w) \omega_{s} d \mu_{O_{t}} .
$$

Here $d \mu_{O_{t}}=d x$ follows $\omega_{s}=\omega_{s}^{\mathrm{d}}$ in (3.7), and $\omega_{s}=\omega_{s}^{\mathrm{b}}$ for $d \mu_{O_{t}}=d S_{x}$. Due to the chain rule

$$
\nabla_{y} \tilde{w}=\left(\nabla \phi_{s}^{-\top} \circ \phi_{s}\right) \nabla\left(\tilde{w} \circ \phi_{s}\right) \quad \text { for } \tilde{w} \in V\left(\Omega_{t+s}\right),
$$

the Jacobian determinants in domain and at the boundary are

$$
\omega_{s}^{\mathrm{d}}:=\operatorname{det}\left(\nabla \phi_{s}\right) \text { in } \Omega_{t}, \quad \omega_{s}^{\mathrm{b}}:=\left|\left(\nabla \phi_{s}^{-\top} \circ \phi_{s}\right) n^{t}\right| \omega_{s}^{\mathrm{d}} \text { at } \partial \Omega_{t}
$$

with the transpose of the inverse ${ }^{-\top}$. However, the state equation (2.5a) is nonlinear. Therefore, to define well an adjoint state (see [43]), after transformation we linearize the resulting relations 
using $|w|^{m-2} w \sim\left|u^{t}\right|^{m-2} w$ at the solution $u^{t}$. In doing so, we look for functions $\left(\tilde{u}^{t+s}, \tilde{p}_{t+s}\right) \in$ $U\left(\Omega_{t}\right)$ which solve perturbed Brinkman-Forchheimer equations in the linearized form:

$$
\begin{aligned}
& \int_{\Omega_{t}}\left(2 \mu E\left(\nabla \phi_{s}^{-\top} \circ \phi_{s}, \tilde{u}^{t+s}\right)\right. E\left(\nabla \phi_{s}^{-\top} \circ \phi_{s}, w\right)+\alpha\left|u^{t}\right|^{m-2}\left(\tilde{u}^{t+s}\right)^{\top} w \\
&\left.-\tilde{p}_{t+s} \operatorname{tr}\left(\left(\nabla \phi_{s}^{-\top} \circ \phi_{s}\right) \nabla w\right)\right) \omega_{s}^{\mathrm{d}} d x=\int_{\Omega_{t}}\left(f \circ \phi_{s}\right)^{\top} w \omega_{s}^{\mathrm{d}} d x, \\
& \int_{\Omega_{t}} \lambda \operatorname{tr}\left(\left(\nabla \phi_{s}^{-\top} \circ \phi_{s}\right) \nabla \tilde{u}^{t+s}\right) \omega_{s}^{\mathrm{d}} d x=0 \quad \text { for all }(w, \lambda) \in U\left(\Omega_{t}\right) .
\end{aligned}
$$

For the derivation, we have used (3.8) and the generalized strain tensor (compare to (2.2b)):

$$
E(M, w):=\frac{1}{2}\left(M \nabla w+\nabla w^{\top} M^{\top}\right) \in \mathbb{R}^{d \times d} \quad \text { for } M \in \mathbb{R}^{d \times d}, w \in V\left(\Omega_{t}\right),
$$

such that $E(I, w)=\varepsilon(w)$ for the $d$-by- $d$ identity matrix $I$. For more details, see [20, 21, 37].

According to (2.17), the perturbed optimal value objective function is introduced as

$$
j(s):=\tilde{\mathscr{J}}\left(s, \tilde{u}^{t+s} ; O_{t}\right) \quad \text { for }\left(\tilde{u}^{t+s}, \tilde{p}_{t+s}\right) \in U\left(\Omega_{t}\right) \text { solving (3.10). }
$$

Following the Lagrange approach, we combine together formulas (3.7), (3.10) within the linearized perturbed Lagrangian $\tilde{\mathscr{L}}: I \times V\left(\Omega_{t}\right) \times U\left(\Omega_{t}\right)^{2} \mapsto \overline{\mathbb{R}}$ as

$$
\begin{aligned}
& \tilde{\mathscr{L}}\left(s, u^{t}, u, p, v, q ; \Omega_{t}\right):=\tilde{\mathscr{J}}\left(s, u ; O_{t}\right)-\int_{\Omega_{t}}\left(2 \mu E\left(\nabla \phi_{s}^{-\top} \circ \phi_{s}, u\right) \cdot E\left(\nabla \phi_{s}^{-\top} \circ \phi_{s}, v\right)\right. \\
& \left.\quad+\left(\alpha\left|u^{t}\right|^{m-2} u-\left(f \circ \phi_{s}\right)\right)^{\top} v-p \operatorname{tr}\left(\left(\nabla \phi_{s}^{-\top} \circ \phi_{s}\right) \nabla v\right)-q \operatorname{tr}\left(\left(\nabla \phi_{s}^{-\top} \circ \phi_{s}\right) \nabla u\right)\right) \omega_{s}^{\mathrm{d}} d x .
\end{aligned}
$$

Then the optimal value in (3.12) can be expressed as $j(s)=l(s)$ with the help of the saddle-point (minimax) problem: find a solution quadruple $\left(\tilde{u}^{t+s}, \tilde{p}_{t+s}, \tilde{v}^{t+s}, \tilde{q}_{t+s}\right) \in U\left(\Omega_{t}\right)^{2}$ such that

$$
\begin{aligned}
\tilde{\mathscr{L}}\left(s, u^{t}, \tilde{u}^{t+s}, \tilde{p}_{t+s}, v, q ; \Omega_{t}\right) & \leq \tilde{\mathscr{L}}\left(s, u^{t}, \tilde{u}^{t+s}, \tilde{p}_{t+s}, \tilde{v}^{t+s}, \tilde{q}_{t+s} ; \Omega_{t}\right)=: l(s) \\
& \leq \tilde{\mathscr{L}}\left(s, u^{t}, u, p, \tilde{v}^{t+s}, \tilde{q}_{t+s} ; \Omega_{t}\right) \quad \text { for all }(u, p, v, q) \in U\left(\Omega_{t}\right)^{2}
\end{aligned}
$$

Following the formalism of [15], we introduce the optimal values:

$$
\begin{aligned}
l_{s}:=\sup _{(v, q) \in U\left(\Omega_{t}\right)} \inf _{(u, p) \in U\left(\Omega_{t}\right)} \tilde{\mathscr{L}}\left(s, u^{t}, u, p, v, q\right) & \\
& \leq \inf _{(u, p) \in U\left(\Omega_{t}\right)} \sup _{(v, q) \in U\left(\Omega_{t}\right)} \tilde{\mathscr{L}}\left(s, u^{t}, u, p, v, q\right)=: l^{s}
\end{aligned}
$$

and the corresponding solution sets:

$$
\begin{gathered}
K^{s}:=\left\{(u, p) \in U\left(\Omega_{t}\right) \mid \sup _{(v, q) \in U\left(\Omega_{t}\right)} \tilde{\mathscr{L}}\left(s, u^{t}, u, p, v, q\right)=l^{s}\right\}, \\
K_{s}:=\left\{(v, q) \in U\left(\Omega_{t}\right) \mid \inf _{(u, p) \in U\left(\Omega_{t}\right)} \tilde{\mathscr{L}}\left(s, u^{t}, u, p, v, q\right)=l_{s}\right\},
\end{gathered}
$$

which determine a multi-valued function $\left[s \rightrightarrows K^{s} \times K_{s}\right]: I \rightrightarrows U\left(\Omega_{t}\right)^{2}$.

Theorem 3.1. Let $u^{t}$ be a solution to (2.5), and the following assumptions hold: 
- $u \mapsto \tilde{J}$ is continuous and has a Gâteaux derivative $\tilde{J}^{\prime} \in V\left(\Omega_{t}\right)^{\star}$ such that

$$
\left\langle\tilde{J}^{\prime}\left(s, u ; O_{t}\right), w\right\rangle_{\Omega_{t}}:=\int_{O_{t}}\left\langle\tilde{\gamma}_{s}^{\prime}(u), w\right\rangle \omega_{s} d \mu_{O_{t}}, \quad\left\langle\tilde{\gamma}_{s}(u), w\right\rangle:=\lim _{r \rightarrow 0} \frac{\tilde{\gamma}_{s}(u+r w)-\tilde{\gamma}_{s}(u)}{r},
$$

where $\langle\cdot, \cdot\rangle_{\Omega_{t}}$ denotes the duality pairing between $V\left(\Omega_{t}\right)$ and its dual space $V\left(\Omega_{t}\right)^{\star}$;

- the objective $\tilde{\mathscr{J}}$ is convex such that

$\left\langle\tilde{\mathscr{J}}^{\prime}\left(s, u ; O_{t}\right), w-u\right\rangle_{\Omega_{t}} \leq \tilde{\mathscr{J}}\left(s, w ; O_{t}\right)-\tilde{\mathscr{J}}\left(s, u ; O_{t}\right) \quad$ for $u, w \in V\left(\Omega_{t}\right), s \in I$.

Then, for every $s \in I$, there exists a state $\left(\tilde{u}^{t+s}, \tilde{p}_{t+s}\right) \in U\left(\Omega_{t}\right)$ solving primal system (3.10), and an adjoint state $\left(\tilde{v}^{t+s}, \tilde{q}_{t+s}\right) \in U\left(\Omega_{t}\right)$ satisfying the adjoint system:

$$
\begin{gathered}
\int_{\Omega_{t}}\left(2 \mu E\left(\nabla \phi_{s}^{-\top} \circ \phi_{s}, \tilde{v}^{t+s}\right) \cdot E\left(\nabla \phi_{s}^{-\top} \circ \phi_{s}, w\right)+\alpha\left|u^{t}\right|^{m-2}\left(\tilde{v}^{t+s}\right)^{\top} w\right. \\
\left.-\tilde{q}_{t+s} \operatorname{tr}\left(\left(\nabla \phi_{s}^{-\top} \circ \phi_{s}\right) \nabla w\right)\right) \omega_{s}^{\mathrm{d}} d x=\int_{O_{t}}\left\langle\tilde{\gamma}_{s}^{\prime}\left(\tilde{u}^{t+s}\right), w\right\rangle \omega_{s} d \mu_{O_{t}}, \\
\int_{\Omega_{t}} \lambda \operatorname{tr}\left(\left(\nabla \phi_{s}^{-\top} \circ \phi_{s}\right) \nabla \tilde{v}^{t+s}\right) \omega_{s}^{\mathrm{d}} d x=0 \quad \text { for all }(w, \lambda) \in U\left(\Omega_{t}\right) .
\end{gathered}
$$

The quadruple $\left(\tilde{u}^{t+s}, \tilde{p}_{t+s}, \tilde{v}^{t+s}, \tilde{q}_{t+s}\right) \in K^{s} \times K_{s}$ implies a saddle point satisfying

$$
l(s)=l_{s}=\tilde{\mathscr{L}}\left(s, u^{t}, \tilde{u}^{t+s}, \tilde{p}_{t+s}, \tilde{v}^{t+s}, \tilde{q}_{t+s} ; \Omega_{t}\right)=l^{s} \quad \text { for } s \in I .
$$

For every $u^{t}$ solving (2.5), the saddle-point is unique.

Proof. We introduce an auxiliary, quadratic functional $\mathscr{E}^{\star}: I \times V\left(\Omega_{t}\right)^{2} \mapsto \overline{\mathbb{R}}$ defined by

$$
\mathscr{E}^{\star}\left(s, u^{t}, w\right):=\frac{1}{2} \int_{\Omega_{t}}\left(2 \mu\left\|E\left(\nabla \phi_{s}^{-\top} \circ \phi_{s}, w\right)\right\|_{\mathrm{F}}^{2}+\alpha\left|u^{t}\right|^{m-2}|w|^{2}\right) \omega_{s}^{\mathrm{d}} d x \quad \text { for } w \in V\left(\Omega_{t}\right),
$$

where $\|\cdot\|_{F}$ stands for Frobenius' matrix norm. It is weakly lower semi-continuous, coercive, and Gâteaux-differentiable. Adding linear terms to $\mathscr{E}^{\star}$ in (3.19), these properties provide an $\operatorname{argument}\left(\tilde{u}^{t+s}, \tilde{p}_{t+s}\right) \in U\left(\Omega_{t}\right)$ of the minimum:

$$
\min _{(v, q) \in U\left(\Omega_{t}\right)}\left\{\mathscr{E}^{\star}\left(s, u^{t}, v\right)-\int_{\Omega_{t}}\left(q \operatorname{tr}\left(\left(\nabla \phi_{s}^{-\top} \circ \phi_{s}\right) \nabla v\right)-\left(f \circ \phi_{s}\right)^{\top} v\right) \omega_{s}^{\mathrm{d}} d x\right\} .
$$

Similarly, using the Gâteaux derivative from (3.16a), there exists an argument $\left(\tilde{v}^{t+s}, \tilde{q}_{t+s}\right) \in$ $U\left(\Omega_{t}\right)$ of the minimum:

$$
\min _{(u, p) \in U\left(\Omega_{t}\right)}\left\{\mathscr{E}^{\star}\left(s, u^{t}, u\right)-\int_{\Omega_{t}} p \operatorname{tr}\left(\left(\nabla \phi_{s}^{-\top} \circ \phi_{s}\right) \nabla u\right) \omega_{s}^{\mathrm{d}} d x-\left\langle\tilde{\mathscr{J}}^{\prime}\left(s, \tilde{u}^{t+s} ; O_{t}\right), u\right\rangle_{\Omega_{t}}\right\} .
$$

Inserting the expression (3.13) into (3.14), the maximization problem

$$
\begin{gathered}
\tilde{\mathscr{J}}\left(s, \tilde{u}^{t+s} ; O_{t}\right)-\int_{\Omega_{t}}\left(2 \mu E\left(\nabla \phi_{s}^{-\top} \circ \phi_{s}, \tilde{u}^{t+s}\right) \cdot E\left(\nabla \phi_{s}^{-\top} \circ \phi_{s}, v\right)\right. \\
\left.+\left(\alpha\left|u^{t}\right|^{m-2} \tilde{u}^{t+s}-\left(f \circ \phi_{s}\right)\right)^{\top} v-\tilde{p}_{t+s} \operatorname{tr}\left(\left(\nabla \phi_{s}^{-\top} \circ \phi_{s}\right) \nabla v\right)-q \operatorname{tr}\left(\left(\nabla \phi_{s}^{-\top} \circ \phi_{s}\right) \nabla \tilde{u}^{t+s}\right)\right) \omega_{s}^{\mathrm{d}} d x \\
\quad \leq \tilde{J}\left(s, \tilde{u}^{t+s} ; O_{t}\right)-\int_{\Omega_{t}}\left(2 \mu E\left(\nabla \phi_{s}^{-\top} \circ \phi_{s}, \tilde{u}^{t+s}\right) \cdot E\left(\nabla \phi_{s}^{-\top} \circ \phi_{s}, \tilde{v}^{t+s}\right)\right. \\
\left.+\left(\left|u^{t}\right|^{m-2} \tilde{u}^{t+s}-\left(f \circ \phi_{s}\right)\right)^{\top} \tilde{v}^{t+s}-\tilde{p}_{t+s} \operatorname{tr}\left(\left(\nabla \phi_{s}^{-\top} \circ \phi_{s}\right) \nabla \tilde{v}^{t+s}\right)-\tilde{q}_{t+s} \operatorname{tr}\left(\left(\nabla \phi_{s}^{-\top} \circ \phi_{s}\right) \nabla \tilde{u}^{t+s}\right)\right) \omega_{s}^{\mathrm{d}} d x
\end{gathered}
$$

after shortening $\tilde{\mathscr{J}}\left(s, \tilde{u}^{t+s} ; O_{t}\right)$, tested with $v=\tilde{v}^{t+s} \pm w$ and $q=\tilde{q}_{t+s} \pm \lambda$ implies equations (3.10). Conversely, relations (3.10) satisfies the former inequality in (3.14) as the equality. 
On the other side, the minimization problem (the latter inequality in (3.14)) reads:

$$
\begin{aligned}
& \tilde{J}\left(s, \tilde{u}^{t+s} ; O_{t}\right)-\int_{\Omega_{t}}\left(2 \mu E\left(\nabla \phi_{s}^{-\top} \circ \phi_{s}, \tilde{u}^{t+s}\right) \cdot E\left(\nabla \phi_{s}^{-\top} \circ \phi_{s}, \tilde{v}^{t+s}\right)\right. \\
& +\left(\alpha\left|u^{t}\right|^{m-2} \tilde{u}^{t+s}-\left(f \circ \phi_{s}\right)\right)^{\top} \tilde{v}^{t+s}-\tilde{p}_{t+s} \operatorname{tr}\left(\left(\nabla \phi_{s}^{-\top} \circ \phi_{s}\right) \nabla \tilde{v}^{t+s}\right) \\
& \left.-\tilde{q}_{t+s} \operatorname{tr}\left(\left(\nabla \phi_{s}^{-\top} \circ \phi_{s}\right) \nabla \tilde{u}^{t+s}\right)\right) \omega_{s}^{\mathrm{d}} d x \\
& \leq \tilde{\mathscr{J}}\left(s, u ; O_{t}\right)-\int_{\Omega_{t}}\left(2 \mu E\left(\nabla \phi_{s}^{-\top} \circ \phi_{s}, u\right) \cdot E\left(\nabla \phi_{s}^{-\top} \circ \phi_{s}, \tilde{v}^{t+s}\right)\right. \\
& \left.+\left(\left|u^{t}\right|^{m-2} u-\left(f \circ \phi_{s}\right)\right)^{\top} \tilde{v}^{t+s}-p \operatorname{tr}\left(\left(\nabla \phi_{s}^{-\top} \circ \phi_{s}\right) \nabla \tilde{v}^{t+s}\right)-\tilde{q}_{t+s} \operatorname{tr}\left(\left(\nabla \phi_{s}^{-\top} \circ \phi_{s}\right) \nabla \tilde{u}^{t+s}\right)\right) \omega_{s}^{\mathrm{d}} d x .
\end{aligned}
$$

After shortening the term $-\tilde{q}_{t+s} \operatorname{tr}\left(\left(\nabla \phi_{s}^{-\top} \circ \phi_{s}\right) \nabla \tilde{u}^{t+s}\right)$, substituting here $u=\tilde{u}^{t+s} \pm r w$ and $p=$ $\tilde{p}_{t+s} \pm \lambda$, dividing by $r$ and passing $r \rightarrow 0$, by the differentiability of $\tilde{J}$ assumed in (3.16a), this leads to the variational equations (3.17). Conversely, the necessary optimality condition (3.17) is sufficient for the minimization in (3.14) provided by the convexity assumption (3.16b). The uniqueness for (3.10), (3.17) is standard.

The definition (3.15) justifies that the saddle-point $\left(\tilde{u}^{t+s}, \tilde{p}_{t+s}, \tilde{v}^{t+s}, \tilde{q}_{t+s}\right)$ belongs to the solution set $K^{s} \times K_{s}$ and satisfies equations in (3.18), which completes the proof.

Based on the identity (3.18), in the next section, we find the shape derivative in (2.18).

\section{SHAPE DifFERENTIABILITY OF THE LAGRANGIAN}

Using the Lagrangian approach, as the main result we prove the differentiability of the shape functional with respect to the linearized perturbation given by the parameter $t+s$ at fixed $t$, when $s \rightarrow 0^{+}$. We start with two auxiliary lemmas.

Lemma 4.1. Under the conditions in Theorem 3.1, let the following assumption hold:

- the function $s \mapsto \tilde{\gamma}_{s}$ from (3.7) is continuously differentiable in I with the derivative

$$
\frac{\partial \tilde{\gamma}_{s}}{\partial s}\left(\left.\Lambda\right|_{t+s}, w\right):=\lim _{r \rightarrow 0} \frac{\tilde{\gamma}_{s+r}(w)-\tilde{\gamma}_{s}(w)}{r} \text { for } w \in V\left(\Omega_{t}\right), s \in I .
$$

Then, the asymptotic expansions as $s \rightarrow 0^{+}$in the first argument of the objective $\tilde{\mathscr{J}}$ in (3.7) and the Lagrange function $\tilde{\mathscr{L}}$ from (3.13) take place:

$$
\begin{gathered}
\tilde{\mathscr{J}}\left(s, u ; \Omega_{t}\right)=\mathscr{J}\left(0, u ; \Omega_{t}\right)+s \frac{\partial \tilde{\mathscr{J}}}{\partial s}\left(r s, u ; \Omega_{t}\right), \quad r \in[0,1], \\
\tilde{\mathscr{L}}\left(s, u^{t}, u, p, v, q ; \Omega_{t}\right)=\mathscr{L}\left(0, u, u^{t}, p, v, q ; \Omega_{t}\right)+s \frac{\partial \tilde{\mathscr{L}}}{\partial s}\left(r s, u^{t}, u, p, v, q ; \Omega_{t}\right),
\end{gathered}
$$

for $(u, p, v, q) \in U\left(\Omega_{t}\right)^{2}$. The partial derivatives $\partial \tilde{\mathscr{J}} / \partial s: I \times V\left(\Omega_{t}\right) \mapsto \overline{\mathbb{R}}$ and $\partial \tilde{\mathscr{L}} / \partial s: I \times$ $V\left(\Omega_{t}\right) \times U\left(\Omega_{t}\right)^{2} \mapsto \overline{\mathbb{R}}$ in (4.2) are continuous functions given analytically by

$$
\frac{\partial \tilde{\mathscr{J}}}{\partial s}\left(s, u ; \Omega_{t}\right):=\int_{O_{t}}\left(\left.\operatorname{div}_{O_{t}} \Lambda\right|_{t+s} \tilde{\gamma}_{s}(u)+\frac{\partial \tilde{\gamma}_{s}}{\partial s}\left(\left.\Lambda\right|_{t+s}, u\right)\right) d \mu_{O_{t}},
$$


where the notation $\operatorname{div} O_{t} \Lambda=\operatorname{div} \Lambda$ when $\omega_{s}=\omega_{s}^{\mathrm{d}}$, and implies the tangential divergence $\operatorname{div}_{O_{t}} \Lambda=$ $\operatorname{div} \Lambda-\left(\nabla \Lambda n^{t}\right)^{\top} n^{t}$ when $\omega_{s}=\omega_{s}^{\mathrm{b}}$ according to (3.9), whereas $\partial \tilde{\mathscr{L}} / \partial s$ is given by

$$
\begin{aligned}
& \frac{\partial \tilde{\mathscr{L}}}{\partial s}\left(s, u^{t}, u, p, v, q ; \Omega_{t}\right):=\frac{\partial \tilde{\mathscr{J}}}{\partial s}\left(s, u ; \Omega_{t}\right)-\int_{\Omega_{t}}\left(\left.\operatorname{div} \Lambda\right|_{t+s} \alpha\left|u^{t}\right|^{m-2} u^{\top} v\right. \\
&+2 \mu\left(\left.\operatorname{div} \Lambda\right|_{t+s} \varepsilon(u) \cdot \varepsilon(v)-\varepsilon(u) \cdot E\left(\left.\nabla \Lambda\right|_{t+s} ^{\top}, v\right)-\varepsilon(v) \cdot E\left(\left.\nabla \Lambda\right|_{t+s} ^{\top}, u\right)\right) \\
&\left.\quad-\left(\left.\operatorname{div} \Lambda\right|_{t+s} f+\left.\nabla f \Lambda\right|_{t+s}\right)^{\top} v+p \operatorname{tr}\left(\left.\nabla \Lambda\right|_{t+s} ^{\top} \nabla v\right)+q \operatorname{tr}\left(\left.\nabla \Lambda\right|_{t+s} ^{\top} \nabla u\right)\right) d x .
\end{aligned}
$$

Proof. Since $s \mapsto \tilde{\mathscr{L}}$ is continuously differentiable, by the mean value theorem, there exists $r \in[0,1]$ such that the asymptotic representation (4.2) holds. We substitute into (3.7), (3.13) the expansion $\phi_{s}=x+\left.s \Lambda\right|_{t+r s}$ as $s \rightarrow 0$ according to (3.3) and (3.5). Together with (4.1), it follows for $u \in V\left(\Omega_{t}\right)$ that (see [3, Chapter 2]):

$$
\begin{array}{r}
f \circ \phi_{s}=f+\left.s \Lambda\right|_{t+r s} ^{\top} \nabla f, \quad \nabla \phi_{s}^{-1} \circ \phi_{s}=I-\left.s \nabla \Lambda\right|_{t+r s}, \quad \tilde{\gamma}_{s}(u)=\gamma(u)+s \frac{\partial \tilde{\gamma}_{s}}{\partial s}\left(\left.\Lambda\right|_{t+r s}, u\right), \\
\omega_{s}^{\mathrm{d}}=1+\left.s \operatorname{div} \Lambda\right|_{t+r s}, \quad \omega_{s}^{\mathrm{b}}=1+s\left(\left.\operatorname{div} \Lambda\right|_{t+r s}-\left(\left.\nabla \Lambda\right|_{t+r s} n^{t}\right)^{\top} n^{t}\right) .
\end{array}
$$

Thus, we derive the partial derivatives $\partial \tilde{\mathscr{J}} / \partial s$ and $\partial \tilde{\mathscr{L}} / \partial s$. This proves the assertion.

The next lemma establishes a sequential semi-continuity property for the solution set $K^{s} \times K_{S}$.

Lemma 4.2. Under the conditions in Lemma 4.1, let the following assumptions hold:

- $\tilde{\gamma}_{s}^{\prime}\left(\tilde{u}^{t+s}\right)$ from (3.16a) on the solutions $\tilde{u}^{t+s}$ to (3.10) is bounded:

$$
\left\|\tilde{\gamma}_{s}\left(\tilde{u}^{t+s}\right)\right\|_{V\left(\Omega_{t}\right)^{\star}} \leq \bar{K}_{\gamma}, \quad \bar{K}_{\gamma} \geq 0
$$

- $s \mapsto \tilde{J}^{\prime}\left(s, \tilde{u}^{t+s} ; O_{t}\right)$ on the solutions is continuous as $s \rightarrow 0^{+}$in the sense:

$$
\text { if } \tilde{u}^{t+s} \rightarrow u^{t} \text { weakly in } V\left(\Omega_{t}\right) \text {, then } \tilde{\gamma}_{s}\left(\tilde{u}^{t+s}\right) \rightarrow \tilde{\gamma}_{0}\left(u^{t}\right) \star \text {-strongly in } V\left(\Omega_{t}\right)^{\star} \text {. }
$$

Then there exists a subsequence $s_{k}$ of saddle-points in (3.18) such that as $s_{k} \rightarrow 0^{+}$:

$$
\left(\tilde{u}^{t+s_{k}}, \tilde{p}_{t+s_{k}}, \tilde{v}^{t+s_{k}}, \tilde{q}_{t+s_{k}}\right) \rightarrow\left(u^{t}, p_{t}, v^{t}, q_{t}\right) \text { strongly in } U\left(\Omega_{t}\right)^{2},
$$

where $\left(u^{t}, p_{t}\right) \in U\left(\Omega_{t}\right)$ solves the unperturbed Brinkman-Forchheimer problem (2.5). Its adjoint state $\left(v^{t}, q_{t}\right) \in U\left(\Omega_{t}\right)$ satisfies the adjoint system:

$$
\begin{gathered}
\int_{\Omega_{t}}\left(2 \mu \varepsilon\left(v^{t}\right) \cdot \varepsilon(w)+\alpha\left|u^{t}\right|^{m-2}\left(v^{t}\right)^{\top} w-q_{t} \operatorname{div}(w)\right) d x=\int_{O_{t}}\left\langle\tilde{\gamma}_{0}^{\prime}\left(u^{t}\right), w\right\rangle d \mu_{O_{t}}, \\
\int_{\Omega_{t}} \lambda \operatorname{div}\left(v^{t}\right) d x=0 \quad \text { for all }(w, \lambda) \in U\left(\Omega_{t}\right),
\end{gathered}
$$

which describes the following governing relations:

$$
\begin{gathered}
-\mu \Delta v^{t}+\alpha\left|u^{t}\right|^{m-2} v^{t}+\nabla q_{t}=\mathbf{1}_{O_{t} \cap \Omega_{t}} \tilde{\gamma}_{0}\left(u^{t}\right), \quad \operatorname{div}\left(v^{t}\right)=0 \quad \text { in } \Omega_{t}, \\
\varepsilon\left(v^{t}\right):=\frac{1}{2}\left(\nabla v^{t}+\left(\nabla v^{t}\right)^{\top}\right), \\
v^{t}=0 \quad \text { on } \Gamma_{t}^{\mathrm{D}}, \quad-2 \mu \varepsilon\left(v^{t}\right) n^{t}+q_{t} n^{t}=\mathbf{1}_{O_{t} \cap \Gamma_{t}^{\mathrm{N}}} \tilde{\gamma}_{0}\left(u^{t}\right) \quad \text { on } \Gamma_{t}^{\mathrm{N}},
\end{gathered}
$$

where the indicator function of a set $A$ is $\mathbf{1}_{A}(x)=1$ if $x \in A$, and zero otherwise.

Proof. We split the proof into weak and strong convergences. 
Weak convergence. We expand the perturbed equation (3.10a) with the help of (4.4) and use the Cauchy-Schwarz inequality to derive for $w \in V\left(\Omega_{t}\right)$ that

$$
\begin{aligned}
\mid \int_{\Omega_{t}}\left(2 \mu \varepsilon\left(\tilde{u}^{t+s}\right) \cdot \varepsilon(w)\right. & \left.+\alpha\left|u^{t}\right|^{m-2}\left(\tilde{u}^{t+s}\right)^{\top} w \omega_{s}^{\mathrm{d}}-\tilde{p}_{t+s} \operatorname{div}(w)-f^{\top} w\right) d x \mid \\
& \leq s C_{0}\left(1+\left\|\tilde{p}_{t+s}\right\|_{L^{2}\left(\Omega_{t}\right)}+\left\|\tilde{u}^{t+s}\right\|_{H^{1}\left(\Omega_{t}\right)^{d}}\right)\|w\|_{H^{1}\left(\Omega_{t}\right)^{d}}, \quad C_{0}>0 .
\end{aligned}
$$

The perturbed incompressibility condition $\operatorname{tr}\left(\left(\nabla \phi_{s}^{-\top} \circ \phi_{s}\right) \nabla \tilde{u}^{t+s}\right) \omega_{s}^{\mathrm{d}}=0$ in (3.10b) implies that $\operatorname{div}\left(\tilde{u}^{t+s}\right)=\mathrm{O}\left(s \nabla \tilde{u}^{t+s}\right)$. Therefore, testing (4.9) with $w=\tilde{u}^{t+s}$, using $\alpha\left|u^{t}\right|^{m-2}\left|\tilde{u}^{t+s}\right|^{2} \omega_{s}^{\mathrm{d}} \geq 0$ due to $\omega_{s}^{\mathrm{d}} \geq 0$ for small $s$, and the Korn-Poincaré inequality (2.7) proceeds with $C_{1} \geq C_{0}$ :

$$
\left\|\tilde{u}^{t+s}\right\|_{H^{1}\left(\Omega_{t}\right)^{d}} \leq \frac{1}{2 \mu K_{\mathrm{KP}}}\left(\|f\|_{L^{2}\left(\Omega_{t}\right)^{d}}+s C_{1}\left(1+\left\|\tilde{p}_{t+s}\right\|_{L^{2}\left(\Omega_{t}\right)}\right)\right)=: C_{u}>0 .
$$

For the pressure, (3.10a) builds a linear bounded functional in $V\left(\Omega_{t}\right)^{\star}$. From (4.10a) and asymptotic formulas (4.4) as $s \rightarrow 0^{+}$, we estimate the following expression from above

$$
\begin{aligned}
& \left|\int_{\Omega_{t}} \tilde{p}_{t+s} \operatorname{tr}\left(\left(\nabla \phi_{s}^{-\top} \circ \phi_{s}\right) \nabla w\right) \omega_{s}^{\mathrm{d}} d x\right| \\
& =\left|\int_{\Omega_{t}}\left(2 \mu E\left(\nabla \phi_{s}^{-\top} \circ \phi_{s}, \tilde{u}^{t+s}\right) \cdot E\left(\nabla \phi_{s}^{-\top} \circ \phi_{s}, w\right)+\left(\alpha\left|u^{t}\right|^{m-2} \tilde{u}^{t+s}-f \circ \phi_{s}\right)^{\top} w\right) \omega_{s}^{\mathrm{d}} d x\right| \\
& \leq C_{2}\left(2 \mu C_{u}+\alpha K_{3}\left\|u^{t}\right\|_{H^{1}\left(\Omega_{t}\right)^{d}} C_{u}+\|f\|_{L^{2}\left(\Omega_{t}\right)^{d}}\right)\|w\|_{H^{1}\left(\Omega_{t}\right)^{d}},
\end{aligned}
$$

where constant $C_{2}>0$, and the Hölder estimate with $K_{3}$ from (2.8) when $m=3$ was used:

$$
\left.\left|\int_{\Omega_{t}}\right| u^{t}\right|^{m-2}\left(\tilde{u}^{t+s}\right)^{\top} w d x \mid \leq K_{3}\left\|u^{t}\right\|_{H^{1}\left(\Omega_{t}\right)^{d}}\left\|\tilde{u}^{t+s}\right\|_{H^{1}\left(\Omega_{t}\right)^{d}}\|w\|_{H^{1}\left(\Omega_{t}\right)^{d}}
$$

Dividing it by the norm $\|w\|_{H^{1}\left(\Omega_{t}\right)^{d}}$ and accounting for the linear dependence of $C_{u}$ on $s \tilde{p}_{t+s}$ in (4.10a) provide the uniform in $s \in I$ upper bound:

$$
\frac{1}{\|w\|_{H^{1}\left(\Omega_{t}\right)^{d}}}\left|\int_{\Omega_{t}} \tilde{p}_{t+s} \operatorname{div}(w) d x\right| \leq C_{3}+s C_{4}\left\|\tilde{p}_{t+s}\right\|_{L^{2}\left(\Omega_{t}\right)}, \quad C_{3}, C_{4}>0 .
$$

Henceforth, for small $s_{0}<K_{\mathrm{LBB}} / C_{4}$, it follows from the LBB condition (2.10) that

$$
\left\|\tilde{p}_{t+s}\right\|_{L^{2}\left(\Omega_{t}\right)} \leq \frac{C_{3}}{K_{\mathrm{LBB}}-s_{0} C_{4}}=: C_{p}>0 .
$$

For adjoint problem (3.17), due to $\omega_{s}^{\mathrm{d}} \leq K_{\omega}, K_{\omega} \geq 1$, and the assumption (4.5a) such that

$$
\int_{O_{t}}\left\langle\tilde{\gamma}_{s}^{\prime}\left(\tilde{u}^{t+s}\right), w\right\rangle d \mu_{O_{t}} \leq\left\|\tilde{\gamma}_{s}\left(\tilde{u}^{t+s}\right)\right\|_{V\left(\Omega_{t}\right)^{\star}}\|w\|_{H^{1}\left(\Omega_{t}\right)^{d}} \leq \bar{K}_{\gamma}\|w\|_{H^{1}\left(\Omega_{t}\right)^{d}}
$$

similarly to (4.10a) and (4.10c), we derive the uniform estimates for $\tilde{v}^{t+s}$ and $\tilde{q}_{t+s}$ :

$$
\left\|\tilde{v}^{t+s}\right\|_{H^{1}\left(\Omega_{t}\right)^{d}} \leq \frac{1}{2 \mu K_{\mathrm{KP}}}\left(\bar{K}_{\gamma} K_{\omega}+s_{0} C_{5}\left(1+\left\|\tilde{q}_{t+s}\right\|_{L^{2}\left(\Omega_{t}\right)}\right)\right)=: C_{v}, \quad\left\|\tilde{q}_{t+s}\right\|_{L^{2}\left(\Omega_{t}\right)} \leq C_{q},
$$

with constant $C_{5}, C_{v}, C_{q}>0$. Based on (4.10) and the compact embedding $H^{1}\left(\Omega_{t}\right) \subset L^{m}\left(\Omega_{t}\right)$, we have that a subsequence $s_{k} \rightarrow 0^{+}$and an accumulation point $\left(u^{t}, p_{t}, v^{t}, q_{t}\right) \in U\left(\Omega_{t}\right)^{2}$ exist with

$$
\begin{aligned}
&\left(\tilde{u}^{t+s_{k}}, \tilde{p}_{t+s_{k}}, \tilde{v}^{t+s_{k}}, \tilde{q}_{t+s_{k}}\right) \rightarrow\left(u^{t}, p_{t}, v^{t}, q_{t}\right) \quad \text { weakly in } U\left(\Omega_{t}\right)^{2} \\
&\left(\tilde{u}^{t+s_{k}}, \tilde{v}^{t+s_{k}}\right) \rightarrow\left(u^{t}, v^{t}\right) \quad \text { strongly in } L^{m}\left(\Omega_{t}\right)^{2 d} .
\end{aligned}
$$


On taking the limit in (3.10) and (3.17) as $s_{k} \rightarrow 0$, in the virtue of convergence (4.11) and the assumption (4.5b), we arrive at unperturbed problem (2.5) and adjoint system (4.7).

Strong convergence. Using the equality $\left|\tilde{u}^{t+s_{k}}-u^{t}\right|^{2}=\left|\tilde{u}^{t+s_{k}}\right|^{2}-\left|u^{t}\right|^{2}-2\left(\tilde{u}^{t+s_{k}}-u^{t}\right)^{\top} u^{t}$ and

$$
\left\|\varepsilon\left(\tilde{u}^{t+s_{k}}-u^{t}\right)\right\|_{\mathrm{F}}^{2}=\left\|\varepsilon\left(\tilde{u}^{t+s_{k}}\right)\right\|_{\mathrm{F}}^{2}-\left\|\varepsilon\left(u^{t}\right)\right\|_{\mathrm{F}}^{2}-2 \varepsilon\left(\tilde{u}^{t+s_{k}}-u^{t}\right) \cdot \varepsilon\left(u^{t}\right),
$$

we subtract (2.5a) tested with $w=u^{t}$ from the inequality (4.9) with $w=\tilde{u}^{t+s_{k}}$ and use (4.10a):

$$
\begin{aligned}
\int_{\Omega_{t}}\left(2 \mu\left\|\varepsilon\left(\tilde{u}^{t+s_{k}}-u^{t}\right)\right\|_{\mathrm{F}}^{2}+\alpha\left|u^{t}\right|^{m-2}\left|\tilde{u}^{t+s_{k}}-u^{t}\right|^{2} \omega_{s_{k}}^{\mathrm{d}}\right) d x \leq \int_{\Omega_{t}}\left(f^{\top}\left(\tilde{u}^{t+s_{k}}-u^{t}\right)\right. \\
\left.\quad-4 \mu \varepsilon\left(\tilde{u}^{t+s_{k}}-u^{t}\right) \cdot \varepsilon\left(u^{t}\right)-2 \alpha\left|u^{t}\right|^{m-2}\left(\tilde{u}^{t+s_{k}}-u^{t}\right)^{\top} u^{t} \omega_{s_{k}}^{\mathrm{d}}\right) d x+s_{k} C_{6}, \quad C_{6}>0 .
\end{aligned}
$$

On taking the limit superior in (4.12) as $s_{k} \rightarrow 0^{+}$due to convergences (4.11) and the KornPoincaré inequality (2.7) provide the strong convergence in (4.6) by the mean of

$$
\underset{s_{k} \rightarrow 0^{+}}{\limsup }\left\|\tilde{u}^{t+s_{k}}-u^{t}\right\|_{H^{1}\left(\Omega_{t}\right)^{d}}^{2} \leq 0 .
$$

For the pressure, we subtract (2.5a) from (4.9), and use (4.10a) and (4.10b) such that

$$
\begin{aligned}
\mid \int_{\Omega_{t}}\left(\left(\tilde{p}_{t+s_{k}}-p_{t}\right) \operatorname{div}(w)-2 \mu \varepsilon\left(\tilde{u}^{t+s_{k}}-u^{t}\right)\right. & \left.\cdot \varepsilon(w)-\alpha\left|u^{t}\right|^{m-2}\left(\tilde{u}^{t+s_{k}}-u^{t}\right)^{\top} w\right) d x \mid \\
& \leq s_{k} C_{7}\left(1+\left\|\tilde{p}_{t+s}\right\|_{L^{2}\left(\Omega_{t}\right)}\right)\|w\|_{H^{1}\left(\Omega_{t}\right)^{d}}, \quad C_{7} \geq C_{0},
\end{aligned}
$$

where the uniform boundedness of $\left\|\tilde{u}^{t+s}\right\|_{H^{1}\left(\Omega_{t}\right)^{d}}$ from (4.10a) was used. Dividing this inequality by the norm of $w$, and applying (4.10c), (4.10b) and the Hölder inequality follow that

$$
\frac{\left|\int_{\Omega_{t}}\left(\tilde{p}_{t+s_{k}}-p_{t}\right) \operatorname{div}(w) d x\right|}{\|w\|_{H^{1}\left(\Omega_{t}\right)^{d}}} \leq\left(2 \mu+\alpha K_{3}\left\|u^{t}\right\|_{H^{1}\left(\Omega_{t}\right)^{d}}\right)\left\|\tilde{u}^{t+s_{k}}-u^{t}\right\|_{H^{1}\left(\Omega_{t}\right)^{d}}+s_{k} C_{7}\left(1+C_{p}\right) .
$$

On taking the limit as $s_{k} \rightarrow 0^{+}$due to (4.13a), the LBB condition (2.8) leads to the upper bound

$$
\limsup _{s_{k} \rightarrow 0^{+}}\left\|\tilde{p}_{t+s_{k}}-p_{t}\right\|_{L^{2}\left(\Omega_{t}\right)} \leq 0 \text {. }
$$

For (3.17), subtracting the adjoint equation (4.7a) tested with $w=v^{t}$ from the perturbed one (3.10a) tested with $w=\tilde{v}^{t+s_{k}}$, and using (4.10d), similarly to (4.12), we get

$$
\begin{aligned}
& \int_{\Omega_{t}}\left(2 \mu\left\|\varepsilon\left(\tilde{v}^{t+s_{k}}-v^{t}\right)\right\|_{\mathrm{F}}^{2}+\alpha\left|u^{t}\right|^{m-2}\left|\tilde{v}^{t+s_{k}}-v^{t}\right|^{2} \omega_{s_{k}}^{\mathrm{d}}\right) d x \\
& \leq \int_{O_{t}}\left(\left\langle\tilde{\gamma}_{s_{k}}\left(\tilde{u}^{t+s_{k}}\right)-\tilde{\gamma}_{0}^{\prime}\left(u^{t}\right), \tilde{v}^{t+s_{k}}\right\rangle+\left\langle\tilde{\gamma}_{0}\left(u^{t}\right), \tilde{v}^{t+s_{k}}-v^{t}\right\rangle\right) d \mu_{O_{t}} \\
& \quad-\int_{\Omega_{t}}\left(4 \mu \varepsilon\left(\tilde{v}^{t+s_{k}}-v^{t}\right) \cdot \varepsilon\left(v^{t}\right)+2 \alpha\left|u^{t}\right|^{m-2}\left(\tilde{v}^{t+s_{k}}-v^{t}\right)^{\top} v^{t} \omega_{s_{k}}^{\mathrm{d}}\right) d x+s_{k} C_{8},
\end{aligned}
$$

where $C_{8}>0$. Subtracting (4.7a) and (3.10a), and using (4.10),

$$
\begin{aligned}
\left|\int_{\Omega_{t}}\left(\tilde{q}_{t+s_{k}}-q_{t}\right) \operatorname{div}(w)\right| \leq & \mid \int_{\Omega_{t}}\left(2 \mu \varepsilon\left(\tilde{v}^{t+s_{k}}-v^{t}\right) \cdot \varepsilon(w)+\alpha\left|u^{t}\right|^{m-2}\left(\tilde{v}^{t+s_{k}}-v^{t}\right)^{\top} w\right) d x \\
& +\int_{O_{t}}\left\langle\tilde{\gamma}_{s_{k}}\left(\tilde{u}^{t+s_{k}}\right)-\tilde{\gamma}_{0}^{\prime}\left(u^{t}\right), w\right\rangle d \mu_{O_{t}} \mid+s_{k} C_{9}\|w\|_{H^{1}\left(\Omega_{t}\right)^{d}}, \quad C_{9}>0 .
\end{aligned}
$$

Therefore, due to (4.11) and (4.5b), we derive the uniform estimates for $\tilde{v}^{t+s}$ and $\tilde{q}_{t+s}$ :

$$
\underset{s_{k} \rightarrow 0^{+}}{\limsup }\left\|\tilde{v}^{t+s_{k}}-v^{t}\right\|_{H^{1}\left(\Omega_{t}\right)^{d}}^{2}+\limsup _{s_{k} \rightarrow 0^{+}}\left\|\tilde{q}_{t+s_{k}}-q_{t}\right\|_{L^{2}\left(\Omega_{t}\right)} \leq 0
$$


which completes the proof of Lemma 4.2.

Below, we establish the main theorem on shape differentiability.

Theorem 4.1. Under the conditions in Lemmas 4.1 and 4.2, the shape derivative in (2.18) exists and expressed explicitly by the partial derivative from (4.3) as follows

$$
\partial_{+} j(0)=\partial_{+} l(0):=\lim _{s \rightarrow 0^{+}} \frac{l(s)-l(0)}{s}=\frac{\partial \tilde{\mathscr{L}}}{\partial s}\left(0, u^{t}, u^{t}, p_{t}, v^{t}, q_{t} ; \Omega_{t}\right),
$$

where $\left(u^{t}, p_{t}\right) \in U\left(\Omega_{t}\right)$ solves unperturbed Brinkman-Forchheimer problem (2.5), and its adjoint state $\left(v^{t}, q_{t}\right) \in U\left(\Omega_{t}\right)$ satisfies adjoint system (4.7).

Proof. According to the identity $j(s)=l(s)$ for $s \in I$ in (3.18), we sketch the proof of the derivative $\partial_{+} l(0)$ following [2, Chapter 10, Theorem 5.1].

We test with $(u, p, v, q)=\left(u^{t}, p_{t}, v^{t}, q_{t}\right) \in K^{0} \times K_{0}$ the minimax inequalities (3.14) as $s=s_{k}$ :

$$
\begin{array}{r}
\tilde{\mathscr{L}}\left(s_{k}, u^{t}, \tilde{u}^{t+s_{k}}, \tilde{p}_{t+s_{k}}, v^{t}, q_{t} ; \Omega_{t}\right) \leq \tilde{\mathscr{L}}\left(s_{k}, u^{t}, \tilde{u}^{t+s_{k}}, \tilde{p}_{t+s_{k}}, \tilde{v}^{t+s_{k}}, \tilde{q}_{t+s_{k}} ; \Omega_{t}\right)=l\left(s_{k}\right) \\
\leq \tilde{\mathscr{L}}\left(s_{k}, u^{t}, u^{t}, p_{t}, \tilde{v}^{t+s_{k}}, \tilde{q}_{t+s_{k}} ; \Omega_{t}\right) .
\end{array}
$$

Also we insert $(u, p, v, q)=\left(\tilde{u}^{t+s_{k}}, \tilde{p}_{t+s_{k}}, \tilde{v}^{t+s_{k}}, \tilde{q}_{t+s_{k}}\right) \in K^{s_{k}} \times K_{s_{k}}$ into (3.14) as $s=0$ :

$$
\begin{aligned}
\tilde{\mathscr{L}}\left(0, u^{t}, u^{t}, p_{t}, \tilde{v}^{t+s_{k}}, \tilde{q}_{t+s_{k}} ; \Omega_{t}\right) \leq \tilde{\mathscr{L}}\left(0, u^{t}, u^{t}, p_{t}, v^{t}, q_{t} ; \Omega_{t}\right) & =l(0) \\
& \leq \tilde{\mathscr{L}}\left(0, u^{t}, \tilde{u}^{t+s_{k}}, \tilde{p}_{t+s_{k}}, v^{t}, q_{t} ; \Omega_{t}\right) .
\end{aligned}
$$

Subtracting $l(0)$ from the left inequality (4.15) and using the right inequality (4.16), applying the mean value theorem with $\underline{\alpha}_{k} \in(0,1)$ leads to the inequalities

$$
\begin{aligned}
\frac{l\left(s_{k}\right)-l(0)}{s_{k}} & \geq \frac{\tilde{\mathscr{L}}\left(s_{k}, u^{t}, \tilde{u}^{t+s_{k}}, \tilde{p}_{t+s_{k}}, v^{t}, q_{t} ; \Omega_{t}\right)-\tilde{\mathscr{L}}\left(0, u^{t}, \tilde{u}^{t+s_{k}}, \tilde{p}_{t+s_{k}}, v^{t}, q_{t} ; \Omega_{t}\right)}{s_{k}} \\
& =\frac{\partial \tilde{\mathscr{L}}}{\partial s}\left(\underline{\alpha}_{k} s_{k}, u^{t}, \tilde{u}^{t+s_{k}}, \tilde{p}_{t+s_{k}}, v^{t}, q_{t} ; \Omega_{t}\right) .
\end{aligned}
$$

On taking the limit inferior as $s_{k} \rightarrow 0^{+}$proceeds with the lower estimate:

$$
\liminf _{s_{k} \rightarrow 0^{+}} \frac{l\left(s_{k}\right)-l(0)}{s_{k}} \geq \frac{\partial \tilde{\mathscr{L}}}{\partial s}\left(0, u^{t}, u^{t}, p_{t}, v^{t}, q_{t} ; \Omega_{t}\right)
$$

On the other side, subtracting $l(0)$ from the right inequality (4.15), and using the left inequality (4.16), the mean value theorem with weights $\bar{\alpha}_{k} \in(0,1)$ provides the upper estimate:

$$
\begin{aligned}
\frac{l\left(s_{k}\right)-l(0)}{s_{k}} & \leq \frac{\tilde{\mathscr{L}}\left(s_{k}, u^{t}, u^{t}, p_{t}, \tilde{v}^{t+s_{k}}, \tilde{q}_{t+s_{k}} ; \Omega_{t}\right)-\tilde{\mathscr{L}}\left(0, u^{t}, u^{t}, p_{t}, \tilde{v}^{t+s_{k}}, \tilde{q}_{t+s_{k}} ; \Omega_{t}\right)}{s_{k}} \\
& =\frac{\partial \tilde{\mathscr{L}}}{\partial s}\left(\bar{\alpha}_{k} s_{k}, u^{t}, u^{t}, q_{t}, \tilde{v}^{t+s_{k}}, \tilde{q}_{t+s_{k}} ; \Omega_{t}\right) .
\end{aligned}
$$

This leads to the limes superior

$$
\limsup _{s_{k} \rightarrow 0^{+}} \frac{l\left(s_{k}\right)-l(0)}{s_{k}} \leq \frac{\partial \tilde{\mathscr{L}}}{\partial s}\left(0, u^{t}, u^{t}, p_{t}, v^{t}, q_{t} ; \Omega_{t}\right),
$$

which together with the lower estimate (4.17a) proves the limit in (4.14). 


\section{HADAMARD FORMULA AND ITS APPLICATIONS}

Provided by smooth solutions to the Brinkman-Forchheimer equation (see [44]), a Hadamard representation of the shape derivative by boundary integrals is presented next.

Theorem 5.1. Under the conditions of Theorem 4.1, let the primal and adjoint solutions of (2.5) and (4.7) be smooth such that $\left(u^{t}, p_{t}, v^{t}, q_{t}\right) \in\left(H^{2}(G)^{d} \times H^{1}(G)\right)^{2}$ in $G \subset \Omega_{t}$. If the kinematic velocity $\Lambda$ is constant outside some domain $G_{t} \subset G$ with $C^{2,0}{ }_{\text {-smooth boundary } \partial G_{t}}$ and outward normal vector $n^{t}$, then the shape derivative (4.14) is expressed equivalently as

$$
\begin{gathered}
\partial_{+} j(0)=J_{G_{t}}(\Lambda)+J_{\partial G_{t}}(\Lambda)+J_{O_{t}}(\Lambda), \quad J_{O_{t}}(\Lambda):=\int_{O_{t}}\left(\operatorname{div}_{O_{t}} \Lambda \tilde{\gamma}_{s}\left(u^{t}\right)+\frac{\partial \tilde{\gamma}_{s}}{\partial s}\left(\Lambda, u^{t}\right)\right) d \mu_{O_{t}}, \\
J_{G_{t}}(\Lambda):=\int_{G_{t}}\left(\Lambda^{\top}\left(\alpha(m-2)\left|u^{t}\right|^{m-4} u^{t}\left(u^{t}\right)^{\top} v^{t}+\mathbf{1}_{O_{t} \cap \Omega_{t}} \tilde{\gamma}_{0}^{\prime}\left(u^{t}\right)\right)\right) d x \\
J_{\partial G_{t}}(\Lambda):=\int_{\partial G_{t}}\left(\left(\Lambda^{\top} n^{t}\right) \mathscr{D}_{1}\left(u^{t}, u^{t}, v^{t}\right)+\Lambda^{\top} \mathscr{D}^{2}\left(u^{t}, p_{t}, v^{t}, q_{t}\right)\right) d S_{x} .
\end{gathered}
$$

The scalar-valued $\mathscr{D}_{1}$ and vector-valued $\mathscr{D}^{2}=\left(\mathscr{D}_{1}^{2}, \ldots, \mathscr{D}_{d}^{2}\right)^{\top}$ expressions in (5.1) are

$$
\begin{aligned}
\mathscr{D}_{1}\left(u^{t}, u, v\right):=f^{\top} v-2 \mu \varepsilon(u) \cdot \varepsilon(v)-\alpha\left|u^{t}\right|^{m-2} u^{\top} v, \\
\mathscr{D}^{2}(u, p, v, q):=\nabla u^{\top}\left(2 \mu \varepsilon(v) n^{t}-q n^{t}\right)+\nabla v^{\top}\left(2 \mu \varepsilon(u) n^{t}-p n^{t}\right) .
\end{aligned}
$$

Proof. Since $\nabla \Lambda=0$ in $\Omega_{t} \backslash G_{t}$ the shape derivative from Theorem 4.1 is expressed with the help of formulas (4.3) and (4.14) as the sum $\partial_{+} j(0)=I_{1}+I_{2}+I_{3}+J_{O_{t}}(\Lambda)$ specified below. Integrating the terms in (4.3) by parts in $G_{t}$ where the solution $\left(u^{t}, p_{t}, v^{t}, q_{t}\right)$ is smooth, we have

$$
\begin{aligned}
I_{1}:= & -\int_{G_{t}}\left(\operatorname{div} \Lambda\left(2 \mu \varepsilon\left(u^{t}\right) \cdot \varepsilon\left(v^{t}\right)+\alpha\left|u^{t}\right|^{m-2}\left(u^{t}\right)^{\top} v^{t}\right)\right. \\
& \left.\left.-2 \mu \varepsilon\left(u^{t}\right) \cdot E\left(\nabla \Lambda, v^{t}\right)-2 \mu \varepsilon\left(v^{t}\right) \cdot E\left(\nabla \Lambda, u^{t}\right)\right)\right) d x \\
= & \int_{G_{t}} \Lambda^{\top}\left(\alpha(m-2)\left|u^{t}\right|^{m-4} u^{t}\left(u^{t}\right)^{\top} v^{t}+\nabla\left(u^{t}\right)^{\top}\left(\alpha\left|u^{t}\right|^{m-2} v^{t}-2 \mu \operatorname{div} \varepsilon\left(v^{t}\right)\right)\right. \\
& \left.+\nabla\left(v^{t}\right)^{\top}\left(\alpha\left|u^{t}\right|^{m-2} u^{t}-2 \mu \operatorname{div} \varepsilon\left(u^{t}\right)\right)\right) d x \\
& -\int_{\partial G_{t}}\left(\left(\Lambda^{\top} n^{t}\right)\left(2 \mu \varepsilon\left(u^{t}\right) \cdot \varepsilon\left(v^{t}\right)+\alpha\left|u^{t}\right|^{m-2}\left(u^{t}\right)^{\top} v^{t}\right)\right. \\
& \left.-2 \mu \Lambda^{\top}\left(\nabla\left(u^{t}\right)^{\top} \varepsilon\left(v^{t}\right)+\nabla\left(v^{t}\right)^{\top} \varepsilon\left(u^{t}\right)\right) n^{t}\right) d S_{x} .
\end{aligned}
$$

Using the incompressibility $\operatorname{div}\left(u^{t}\right)=\operatorname{div}\left(v^{t}\right)=0$, we have

$$
\begin{aligned}
I_{2} & :=-\int_{G_{t}}\left(p_{t} \operatorname{tr}\left(\nabla \Lambda^{\top} \nabla v^{t}\right)+q_{t} \operatorname{tr}\left(\nabla \Lambda^{\top} \nabla u^{t}\right)\right) d x \\
& =\int_{G_{t}} \Lambda^{\top}\left(\nabla\left(v^{t}\right)^{\top} \nabla p_{t}+\nabla\left(u^{t}\right)^{\top} \nabla q_{t}\right) d x-\int_{\partial G_{t}} \Lambda^{\top}\left(\nabla\left(v^{t}\right)^{\top} p_{t}+\nabla\left(u^{t}\right)^{\top} q_{t}\right) n^{t} d S_{x},
\end{aligned}
$$

and

$$
I_{3}:=\int_{G_{t}}(\operatorname{div} \Lambda f+\nabla f \Lambda)^{\top} v^{t} d x
$$


Employing the identity $\operatorname{div}\left(\Lambda\left(f^{\top} v^{t}\right)\right)=\operatorname{div} \Lambda\left(f^{\top} v^{t}\right)+\Lambda^{\top} \nabla\left(f^{\top} v^{t}\right)$ and equilibrium equations (2.2a), (4.8a), we have

$$
\begin{aligned}
I_{1}+I_{2}+I_{3}= & \int_{G_{t}}\left(\operatorname{div}\left(\Lambda\left(f^{\top} v^{t}\right)\right)+\Lambda^{\top}\left(\alpha(m-2)\left|u^{t}\right|^{m-4} u^{t}\left(u^{t}\right)^{\top} v^{t}+\mathbf{1}_{O_{t} \cap \Omega_{t}} \tilde{\gamma}_{0}^{\prime}\left(u^{t}\right)\right)\right) d x \\
& -\int_{\partial G_{t}}\left(\left(\Lambda^{\top} n^{t}\right)\left(2 \mu \varepsilon\left(u^{t}\right) \cdot \varepsilon\left(v^{t}\right)+\alpha\left|u^{t}\right|^{m-2}\left(u^{t}\right)^{\top} v^{t}\right)\right. \\
& \left.-\Lambda^{\top}\left(\nabla\left(u^{t}\right)^{\top}\left(2 \mu \varepsilon\left(v^{t}\right)-q_{t}\right)+\nabla\left(v^{t}\right)^{\top}\left(2 \mu \varepsilon\left(u^{t}\right)-p_{t}\right)\right) n^{t}\right) d S_{x} \\
= & J_{G_{t}}(\Lambda)+J_{\partial G_{t}}(\Lambda) .
\end{aligned}
$$

The divergence theorem applied to the first term in the integral over $G_{t}$ in the right-hand side follows the formulas for $J_{G_{t}}, \mathscr{D}_{1}$ and $\mathscr{D}^{2}$ in (5.1) and (5.2).

The first corollary of the Hadamard representation concerns path-independent integrals widely used in fracture mechanics (see [19]).

Corollary 5.1. Under the conditions of Theorem 5.1, let the kinematic velocity $\Lambda=0$ in $O_{t}$, and either $\alpha=0$ or $m=2$. Then the Hadamard formula (5.1) implies a path-independent integral

$$
J_{\partial \tilde{G}_{t}}(\Lambda)=J_{\partial G_{t}}(\Lambda):=\int_{\partial G_{t}}\left(\left(\Lambda^{\top} n^{t}\right) \mathscr{D}_{1}\left(u^{t}, u^{t}, v^{t}\right)+\Lambda^{\top} \mathscr{D}^{2}\left(u^{t}, p_{t}, v^{t}, q_{t}\right)\right) d S_{x},
$$

which is constant over $C^{2,0}$-smooth boundaries $\partial \tilde{G}_{t}$ of domains $\tilde{G}_{t}$ such that $G_{t} \subset \tilde{G}_{t} \subset G \subset \Omega_{t}$.

Proof. The representation (5.3) is obtained straightforwardly from (5.1) when $J_{G_{t}}(\Lambda)=J_{O_{t}}(\Lambda)=$ 0 , and its constant value is provided by the uniqueness of the shape derivative in (4.14).

We decompose vectors into orthogonal, normal and tangential components at the boundary:

$$
\Lambda=\left(\Lambda^{\top} n^{t}\right) n^{t}+\Lambda_{\tau^{t}}, \quad \mathscr{D}^{2}=\left(\left(\mathscr{D}^{2}\right)^{\top} n^{t}\right) n^{t}+\mathscr{D}_{\tau^{t}}^{2} .
$$

Corollary 5.2. Under the conditions of Corollary 5.1, let the kinematic velocity $\Lambda^{\top} n^{t}=0$ at $\partial \Omega_{t}$. Then the path-independent integral (5.3) implies that

$$
J_{\partial G_{t}}(\Lambda)=J_{\partial G_{t} \cap \Omega_{t}}(\Lambda) .
$$

If the solutions are smooth in the whole $\Omega_{t}$ such that $\left(u^{t}, p_{t}, v^{t}, q_{t}\right) \in\left(H^{2}\left(\Omega_{t}\right)^{d} \times H^{1}\left(\Omega_{t}\right)\right)^{2}$, then $J_{\partial \Omega_{t}}(\Lambda)=0$ for all $\Lambda$ such that $\Lambda=0$ in $O_{t}$ and $\Lambda^{\top} n^{t}=0$ at $\partial \Omega_{t}$.

Proof. We split $\partial G_{t}$ into the part $\partial G_{t} \cap \Omega_{t}$ inside $\Omega_{t}$ and two parts meeting either the Dirichlet $\partial G_{t} \cap \Gamma_{t}^{\mathrm{D}}$ or the Neumann $\partial G_{t} \cap \Gamma_{t}^{\mathrm{N}}$ boundaries, respectively. Since $\Lambda^{\top} n^{t}=0$ is assumed at $\partial \Omega_{t}$, it follows from (5.3) and (5.4) that the decomposition

$$
\begin{aligned}
J_{\partial G_{t}}(\Lambda)=\int_{\partial G_{t} \cap \Omega_{t}} & \left(\left(\Lambda^{\top} n^{t}\right) \mathscr{D}_{1}\left(u^{t}, u^{t}, v^{t}\right)+\Lambda^{\top} \mathscr{D}^{2}\left(u^{t}, p_{t}, v^{t}, q_{t}\right)\right) d S_{x} \\
& +\int_{\partial G_{t} \cap \Gamma_{t}^{\mathrm{D}}} \Lambda_{\tau^{t}}^{\top} \mathscr{D}^{2}\left(u^{t}, p_{t}, v^{t}, q_{t}\right)_{\tau^{t}} d S_{x}+\int_{\partial G_{t} \cap \Gamma_{t}^{\mathrm{N}}} \Lambda_{\tau^{t}}^{\top} \mathscr{D}^{2}\left(u^{t}, p_{t}, v^{t}, q_{t}\right)_{\tau^{t}} d S_{x} .
\end{aligned}
$$

At $\Gamma_{t}^{\mathrm{D}}$, it holds $\left(\nabla u_{1}^{t}\right)_{\tau^{t}}=\ldots=\left(\nabla u_{d}^{t}\right)_{\tau^{t}}=0$ and $\left(\nabla v_{1}^{t}\right)_{\tau^{t}}=\ldots=\left(\nabla v_{d}^{t}\right)_{\tau^{t}}=0$ due to the homogeneous Dirichlet conditions in (2.2c) and (4.8c). Henceforth, according to formula (5.2), $\mathscr{D}^{2}\left(u^{t}, p_{t}, v^{t}, q_{t}\right)_{\tau^{t}}=0$ at $\Gamma_{t}^{\mathrm{D}}$. The substitution of Neumann boundary conditions (2.2c) and (4.8c) into $\mathscr{D}^{2}\left(u^{t}, p_{t}, v^{t}, q_{t}\right)$ eliminates its contribution at $\Gamma_{t}^{\mathrm{N}}$. Thus, we arrive at (5.5).

If it is possible to take $G_{t}=\Omega_{t}$, then the integral in (5.5) is trivial in this case. 
It is worth noting that, in general, the singularity happens at the intersection $\overline{\Gamma_{t}^{D}} \cap \overline{\Gamma_{t}^{N}}$. For illustration, in Figure 1, we present an example geometry of the cracked domain in $2 \mathrm{~d}$ :

$$
\begin{aligned}
& \left.\left.\Omega_{t}=\left\{x_{1} \in(-2,2), x_{2} \in(-1,1)\right)\right\} \backslash\left\{x_{1}=0, x_{2} \in(0,1)\right)\right\}, \\
& \Gamma_{t}^{\mathrm{D}}=\partial \Omega_{t} \cap\left\{x_{2}<0\right\}, \quad \Gamma_{t}^{\mathrm{N}}=\partial \Omega_{t} \cap\left\{x_{2}>0\right\}, \quad O_{t}=\partial \Omega_{t} \cap\left\{x_{2}=1\right\} .
\end{aligned}
$$

It has three singular points: the crack tip $P_{0}=(0,0)$, and $P_{ \pm}=( \pm 1,0)$ where the Dirichlet

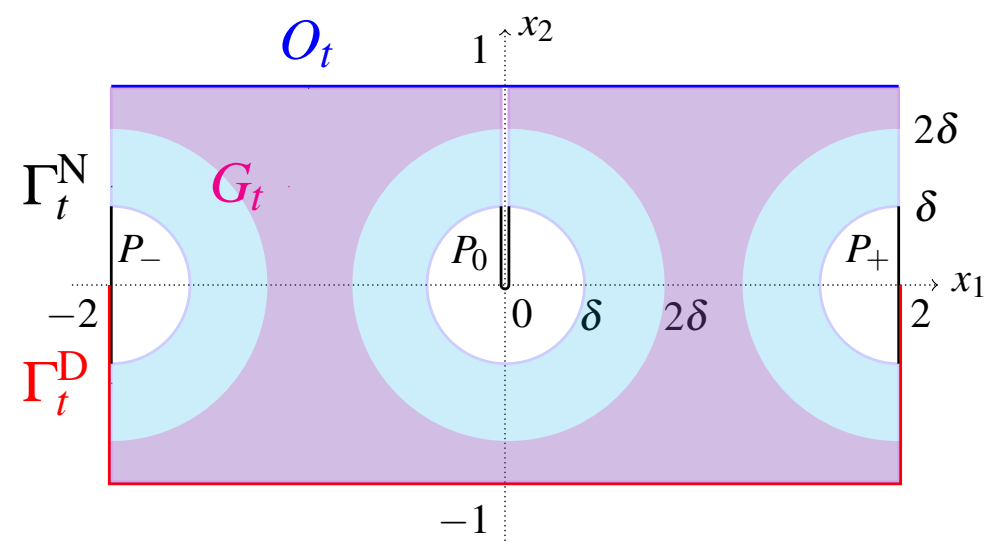

FIGURE 1. Example geometry $\Omega_{t}$ in $2 \mathrm{~d}$.

$\Gamma_{t}^{\mathrm{D}}$ and the Neumann $\Gamma_{t}^{\mathrm{N}}$ boundaries meet each other. We denote by $B_{\delta}(P)$ a circle of radius $\delta \in\left(\delta_{0}, \delta_{1}\right)$ with $0<\delta_{0}<\delta_{1}<1 / 2$, centered at point $P$.

According to the local regularity results well known for the linear elliptic problems, the solution $\left(u^{t}, p_{t}, v^{t}, q_{t}\right)$ is $H^{2} \times H^{1} \times H^{2} \times H^{1}$-smooth in the domain

$$
G=\Omega_{t} \backslash\left(B_{\delta_{0}}\left(P_{0}\right) \cup B_{\delta_{0}}\left(P_{ \pm}\right)\right)
$$

Then admissible domains in Theorem 5.1 and its Corollary 5.1 are, e.g.,

$$
G_{t}=\Omega_{t} \backslash\left(B_{\delta}\left(P_{0}\right) \cup B_{\delta}\left(P_{ \pm}\right)\right) \subset G
$$

for arbitrary $\delta \in\left(\delta_{0}, \delta_{1}\right)$. Respectively, in Corollary 5.2, admissible velocities are $\Lambda_{1}(x) \equiv 0$,

$$
\Lambda_{2}(x)= \begin{cases}k_{0} \text { in } B_{\delta}\left(P_{0}\right), & k_{ \pm} \text {in } B_{\delta}\left(P_{ \pm}\right) ; \\ k_{0}\left(2-\frac{\left|x-P_{0}\right|}{\delta}\right) \text { in } B_{2 \delta}\left(P_{0}\right) \backslash B_{\delta}\left(P_{0}\right), & k_{ \pm}\left(2-\frac{\left|x-P_{ \pm}\right|}{\delta}\right) \text { in } B_{2 \delta}\left(P_{ \pm}\right) \backslash B_{\delta}\left(P_{ \pm}\right) ; \\ 0 \text { in } \Omega_{t} \backslash\left(B_{2 \delta}\left(P_{0}\right) \cup B_{2 \delta}\left(P_{ \pm}\right)\right) ; & \end{cases}
$$

with three arbitrary parameters $k_{0}, k_{ \pm} \in \mathbb{R}$. In this case, we have

$$
\partial G_{t} \cap \Omega_{t}=\left(\partial B_{\delta}\left(P_{0}\right) \cup \partial B_{\delta}\left(P_{ \pm}\right)\right) \cap \Omega_{t} .
$$

This geometric illustration might be helpful for further applications to singular problems.

The last corollary deals with the inverse problem of shape optimal control (2.17), and according to (2.18) it guarantees the descent direction for optimization (see, e.g., [27]). 
Corollary 5.3. Under the conditions of Corollary 5.1, a descent direction $\partial_{+} j(0)<0$ for (2.17) is provided by kinematic velocities $\Lambda$ such that at $\partial G_{t}$ :

$$
\Lambda^{\top} n^{t}=-k_{1}\left(\mathscr{D}_{1}\left(u^{t}, u^{t}, v^{t}\right)+\mathscr{D}^{2}\left(u^{t}, p_{t}, v^{t}, q_{t}\right)^{\top} n^{t}\right), \quad \Lambda_{\tau_{t}}=-k_{2} \mathscr{D}^{2}\left(u^{t}, p_{t}, v^{t}, q_{t}\right) \tau^{t}
$$

with free parameters $k_{1}, k_{2} \geq 0$ such that $k_{1}^{2}+k_{2}^{2} \neq 0$.

Indeed, direct substitution into (5.1) of $\Lambda$ from (5.6) yields

$\partial_{+} j(0)=-\int_{\partial G_{t}}\left(k_{1}\left(\mathscr{D}_{1}\left(u^{t}, u^{t}, v^{t}\right)+\mathscr{D}^{2}\left(u^{t}, p_{t}, v^{t}, q_{t}\right)^{\top} n^{t}\right)^{2}+k_{2}\left|\mathscr{D}^{2}\left(u^{t}, p_{t}, v^{t}, q_{t}\right) \tau^{t}\right|^{2}\right) d S_{x}<0$.

Corollary 5.3 gives practical formulas for numerical simulation by gradient methods.

\section{Acknowledgments}

V.A. Kovtunenko was supported by the Austrian Science Fund (FWF) project P26147-N26: PION and the European Research Council (ERC) under the European Union's Horizon 2020 Research and Innovation Programme (advanced grant No. 668998 OCLOC). V.A.K. thanks the Russian Foundation for Basic Research (RFBR) project 18-29-10007 for partial support.

\section{REFERENCES}

[1] P. Forchheimer, Hydraulik, Teubner, Berlin, 1930.

[2] M.C. Delfour, J.-P. Zolésio, Shape and Geometries: Metrics, Analysis, Differential Calculus, and Optimization, SIAM, Philadelphia, 2011.

[3] J. Sokołowski, J.-P. Zolesio, Introduction to Shape Optimization. Shape Sensitivity Analysis, Springer, Berlin, 1992.

[4] M. Hintermüller, T. Surowiec, First-order optimality conditions for elliptic mathematical programs with equilibrium constraints via variational analysis, SIAM J. Optim. 21 (2011), 1561-1593.

[5] J. Gwinner, B. Jadamba, A.A. Khan, M. Sama, Identification in variational and quasi-variational inequalities, J. Convex Anal. 25 (2018), 545-569.

[6] J. Gwinner, On two-coefficient identification in elliptic variational inequalities, Optimization 67 (2018), 1017-1030.

[7] S.D. Zeng, S. Migorski, A.A. Khan, Nonlinear quasi-hemivariational inequalities: existence and optimal control, SIAM J. Control Optim. 59 (2021), 1246-1274.

[8] J. Gwinner, E.P. Stephan, Advanced Boundary Element Methods: Treatment of Boundary Value, Transmission and Contact Problems, Springer, Berlin, 2018.

[9] T. Apel, D. Sirch, A priori mesh grading for distributed optimal control problems, In: G. Leugering, S. Engell, A. Griewank, M. Hinze, R. Rannacher, V. Schulz, M. Ulbrich, S. Ulbrich, (eds.) Constrained Optimization and Optimal Control for Partial Differential Equations, vol. 160, pp. 377-389, Springer, Basel, 2012.

[10] M. Gerdts, Optimal Control of ODEs and DAEs, De Gruyter, Berlin, 2012.

[11] F. Cakoni, V.A. Kovtunenko, Topological optimality condition for the identification of the center of an inhomogeneity, Inverse Probl. 34 (2018), Article ID 035009.

[12] A. Hasanov, O. Baysal, H. Itou, Identification of an unknown shear force in a cantilever Euler-Bernoulli beam from measured boundary bending moment, J. Inv. Ill-Posed Probl. 27 (2019), 859-876.

[13] V.A. Kovtunenko, K. Kunisch, High precision identification of an object: Optimality-conditions-based concept of imaging, SIAM J. Control Optim. 52 (2014), 773-796.

[14] M.M. Lavrentiev, A.V. Avdeev, M.M. Lavrentiev, Jr., V.I. Priimenko, Inverse Problems of Mathematical Physics, de Gruyter, Berlin, 2012.

[15] R. Correa, A. Seeger, Directional derivative of a minimax function, Nonlinear Anal. 9 (1985), 834-862.

[16] M. Hintermüller, V.A. Kovtunenko, From shape variation to topology changes in constrained minimization: a velocity method-based concept, Optimization Meth. Softw. 26 (2011), 513-532.

[17] K. Ito, K. Kunisch, G. Peichl, Variational approach to shape derivatives, ESAIM: COCV 14 (2008), 517-539. 
[18] S. Schmidt, V. Schulz, Shape derivatives for general objective functions and the incompressible NavierStokes equations, Control Cybernet. 39 (2010), 677-713.

[19] A.M. Khludnev, V.A. Kovtunenko, Analysis of Cracks in Solids, WIT-Press, Southampton, 2000.

[20] V.A. Kovtunenko, Primal-dual methods of shape sensitivity analysis for curvilinear cracks with nonpenetration, IMA J. Appl. Math. 71 (2006), 635-657.

[21] V.A. Kovtunenko, K. Kunisch, Problem of crack perturbation based on level sets and velocities, Z. Angew. Math. Mech. 87 (2007), 809-830.

[22] V.A. Kovtunenko, K. Kunisch, W. Ring, Propagation and bifurcation of cracks based on implicit surfaces and discontinuous velocities, Comput. Visual Sci. 12 (2009), 397-408.

[23] A.M. Khludnev, Inverse problem for elastic body with thin elastic inclusion, J. Inv. Ill-Posed Probl. 28 (2019), 195-209.

[24] V.A. Kovtunenko, G. Leugering, A shape-topological control problem for nonlinear crack - defect interaction: the anti-plane variational model, SIAM J. Control. Optim. 54 (2016), 1329-1351.

[25] N.P. Lazarev, E. M. Rudoy, Optimal size of a rigid thin stiffener reinforcing an elastic plate on the outer edge: Optimal size of a rigid thin stiffener reinforcing an Kirchhoff-Love plate on the outer edge, Z. Angew. Math. Mech. 97 (2017), 1120-1127.

[26] V.V. Shcherbakov, Choosing an optimal shape of thin rigid inclusions in elastic bodies, J. Appl. Mech Tech. Phys. 56 (2015), 321-329.

[27] D. Ghilli, K. Kunisch, V.A. Kovtunenko, Inverse problem of breaking line identification by shape optimization, J. Inv. Ill-Posed Probl. 28 (2020), 119-135.

[28] O.A. Ladyzhenskaya, The Mathematical Theory of Viscous Incompressible Flow, Science Publishers, New York, 1969.

[29] D. Nield, A. Bejan, Convection in Porous Media, Springer, New York, 2006.

[30] K.R. Rajagopal, On a hierarchy of approximate models for flows of incompressible fluids through porous solids, Math. Mod. Meth. Appl. Sci. 17 (2007), 215-252.

[31] P.V. Kovtunenko, Perturbation propagation in a thin layer of a viscosity-stratified fluid, J. Math. Sci. 215 (2016), 499-509.

[32] J.C. de los Reyes, F. Tröltzsch, Optimal control of the stationary Navier-Stokes equations with mixed controlstate constraints, SIAM J. Control Optim. 46 (2007), 604-629.

[33] F. Bouchon, G.H. Peichl, M. Sayeh, R. Touzani, A free boundary problem for the Stokes equations, ESAIM: COCV 23 (2017), 195-215.

[34] G.V. Alekseev, D.A. Tereshko, Numerical analysis of control problems for stationary models of hydrodynamics and heat transfer, In: H. Choi, H.G. Choi, J.Y. Yoo, (eds.) Computational Fluid Dynamics, pp. 335-340, Springer, Berlin, 2008.

[35] J. Haslinger, R.A.E. Mäkinen, The parameter identification in the Stokes system with threshold slip boundary conditions, Z. Angew. Math. Mech. 59 (2020), doi: 10.1002/zamm.201900209.

[36] P.I. Plotnikov, J. Sokolowski, Compressible Navier-Stokes Equations. Theory and Shape Optimization, Birkhüser, Basel, 2012.

[37] V.A. Kovtunenko, K. Ohtsuka, Shape differentiability of Lagrangians and application to Stokes problem, SIAM J. Control Optim. 56 (2018), 3668-3684.

[38] J.R. González Granada, J. Gwinner, V.A. Kovtunenko, On the shape differentiability of objectives: a Lagrangian approach and the Brinkman problem, Axioms 7 (2018), Article ID 76.

[39] M. Bulíček, J. Málek, J. Žabenský, A generalization of the Darcy-Forchheimer equation involving an implicit, pressure-dependent relation between the drag force and the velocity, J. Math. Anal. Appl. 424 (2015), 785-801.

[40] J.K. Djoko, P.A. Razafimandimby, Analysis of the Brinkman-Forchheimer equations with slip boundary conditions, Applicable Anal. 93 (2014), 1477-1494.

[41] P. Skrzypacz, D. Wei, Solvability of the Brinkman-Forchheimer-Darcy equation, J. Appl. Math. 56 (2017), Article ID 7305230.

[42] M. Kohr, M. Lanza de Cristoforis, S.E. Mikhailov, W.L. Wendland, Integral potential method for transmission problem with Lipschitz interface in $\mathbb{R}^{3}$ for the Stokes and Darcy-Forchheimer-Brinkman PDE systems, Z. Angew. Math. Phys. 67 (2016), Article ID 116. 
[43] G.I. Marchuk, V.I. Agoshkov, V.P. Shutyaev, Adjoint Equations and Perturbation Algorithms in Nonlinear Problems, CRC Press, Boca Raton, 1996.

[44] P. Fabrie, Regularity of the solution of Darcy-Forchheimer's equation, Nonlinear Anal. 13 (1989), 10251049. 